Document downloaded from:

http://hdl.handle.net/10251/159838

This paper must be cited as:

Zafar, F.; Cordero Barbero, A.; Torregrosa Sánchez, JR. (2019). Stability analysis of a family of optimal fourth-order methods for multiple roots. Numerical Algorithms. 81(3):947-981. https://doi.org/10.1007/s11075-018-0577-0

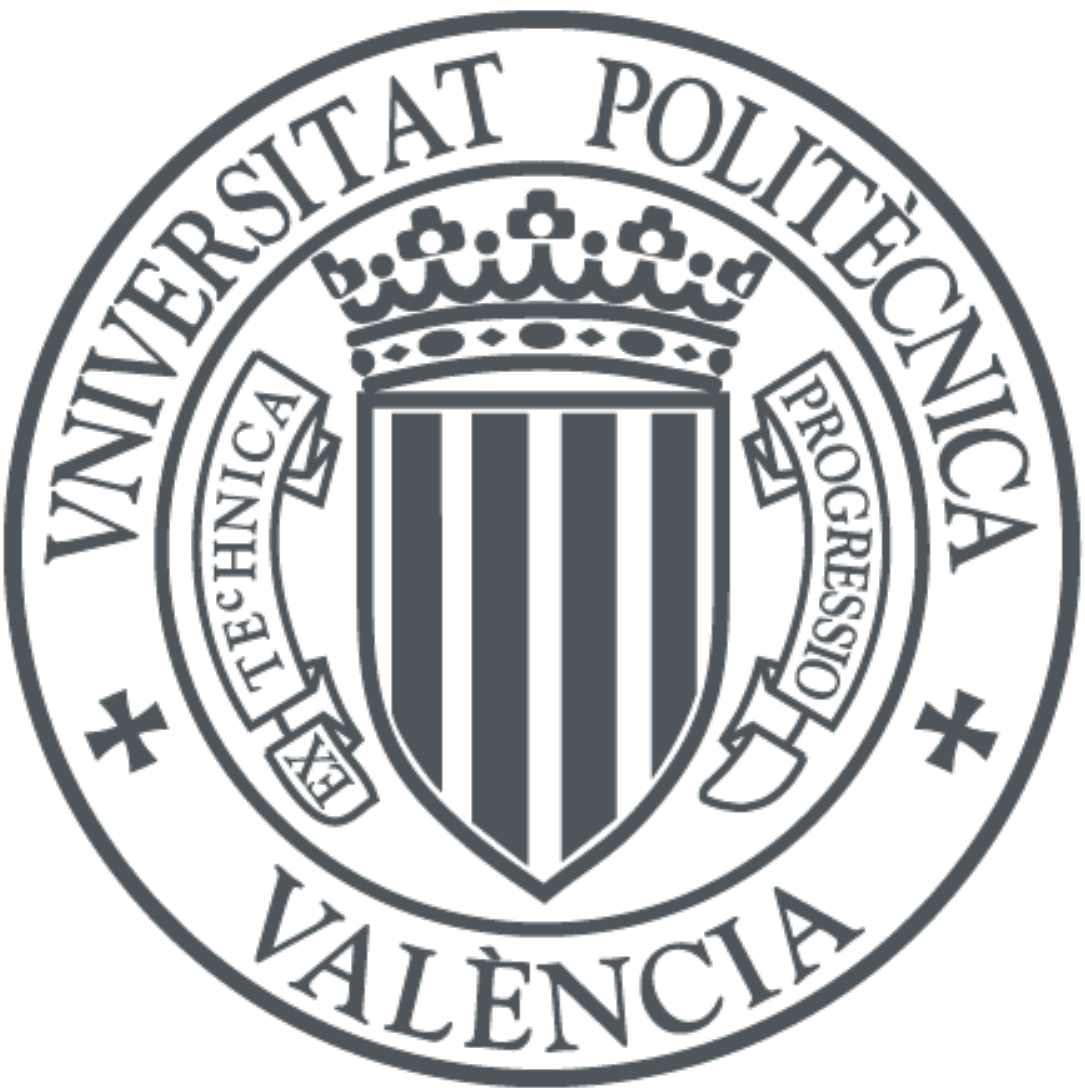

The final publication is available at

https://doi.org/10.1007/s11075-018-0577-0

Copyright Springer-Verlag

Additional Information 


\title{
Stability Analysis of a Family of Optimal Fourth Order Methods for Multiple roots
}

\author{
Fiza Zafar • Alicia Cordero · Juan R. Torregrosa
}

Received: date / Accepted: date

\begin{abstract}
Complex dynamics tools applied on the rational functions resulting from a parametric family of roots solvers for nonlinear equations provide very useful results that have been stated in the last years. These qualitative properties allow the user to select the most efficient members from the family of iterative schemes, in terms of stability and wideness of the sets of convergent initial guesses. These tools have been widely used in the case of iterative procedures for finding simple roots and only recently are being applied on the case of multiplicity $m>1$. In this paper, by using weight function procedure, we design a general class of iterative methods for calculating multiple roots that includes some known methods. In this class, conditions on the weight function are not very restrictive, so a large number of different subfamilies can be generated, all of them are optimal with fourth-order of convergence. Their dynamical analysis give us enough information to select those with better properties and test them on different numerical experiments, showing their numerical properties.
\end{abstract}

Keywords Nonlinear equations $\cdot$ Multiple zeros $\cdot$ Optimal methods $\cdot$ Weight functions $\cdot$ Complex dynamics $\cdot$ Parameter and dynamical planes

\section{Introduction}

Let $f: \mathbb{C} \rightarrow \mathbb{C}$ have a multiple zero $\xi$ with known integer multiplicity $m>1$ and let $f$ be analytic in a small neighborhood of $\xi$. Finding a repeated zero for such a function $f$ has always been of much interest and attention due to its wide applications in various fields of science and engineering; see, for example, the case of continuous stirred tank reactor in [1], fractional conversion problem in a chemical reactor [2], or the multipactor problem [3,4].

The modified Newton's method is usually applied to solve this problem. Keeping in view that this modified method for multiple roots is a quadratically convergent, one-point optimal method, there has always remained a need to define higherorder multipoint iterative schemes to significantly apply to more complicated problems of real world. In the last decade, some efforts have been made to give optimal and non-optimal fourth order convergent methods by Neta and Johnson [5] in 2008, Li et al. [6] in 2009, Neta [7], Sharma and Sharma [8] and Li et al. [9] in 2010, Zhou et al. [10] in 2011, Sharifi et al. [11] in 2012, Soleymani et al. [12], Soleymani and Babajee [13], Liu and Zhou [14] and Zhou et al. [15] in 2013, Thukral [16] in 2014, Behl et al. [17] and Hueso et al. [3] in 2015, Behl et al. [18] in 2016 and Lee et al. [19] in 2017.

This research was partially supported by Ministerio de Economía y Competitividad MTM2014-52016-C2-2-P, Generalitat Valenciana PROMETEO/2016/089 and Schlumberger Foundation-Faculty for Future Program.

Fiza Zafar

Centre for Advanced Studies in Pure and Applied Mathematics,

Bahauddin Zakariya University, Multan 60800, Pakistan E-mail: fizazafar@gmail.com

Alicia Cordero

Instituto Universitario de Matemática Multidisciplinar

Universitat Politècnica de València, 46022 València, Spain

E-mail: acordero@mat.upv.es

Juan R.Torregrosa

Instituto Universitario de Matemática Multidisciplinar

Universitat Politècnica de València, 46022 València, Spain

E-mail: jrtorre@mat.upv.es 
Among these methods, much attention have been given to Jarratt type schemes, however, Zhou et al. [15] proposed an optimal fourth order method for multiple roots involving two function and a derivative evaluation as follows:

$$
\begin{aligned}
y_{n} & =x_{n}-m \frac{f\left(x_{n}\right)}{f^{\prime}\left(x_{n}\right)}, \\
x_{n+1} & =x_{n}-m G(v) \frac{f\left(x_{n}\right)}{f^{\prime}\left(x_{n}\right)},
\end{aligned}
$$

where $v=\left(\frac{f\left(y_{n}\right)}{f\left(x_{n}\right)}\right)^{\frac{1}{m}}$ and $G(\cdot) \in \mathcal{C}^{2}(R)$ is a weight function.

Many authors have also developed optimal fourth order methods involving free parameters as follows: In 2015 , Behl et al. in [17] presented an optimal fourth-order convergent iterative method for investigating multiple roots of nonlinear equations by using five parameters as given below:

$$
\begin{aligned}
y_{n} & =x_{n}-\frac{2 m}{m+2} \frac{f\left(x_{n}\right)}{f^{\prime}\left(x_{n}\right)}, \\
x_{n+1} & =x_{n}-\left[\frac{2 m a_{1} f\left(x_{n}\right)}{(3 m-2) f^{\prime}\left(x_{n}\right)+(m+2) a_{2} f^{\prime}\left(y_{n}\right)}+\frac{m a_{3} f\left(x_{n}\right)}{(m-2) a_{4} f^{\prime}\left(x_{n}\right)+(m+2) a_{5} f^{\prime}\left(y_{n}\right)}\right],
\end{aligned}
$$

where $a_{1}, a_{2}, a_{3}, a_{4}$, and $a_{5}$ are real parameters in which $a_{4}$ and $a_{5}$ are free parameters and others are depending on $m$ as given below:

$a_{1}=\frac{-(3 m-2)\left(m^{2}(m-2) a_{4}+u\left(m^{3}+4 m^{2}-8\right) a_{5}\right)^{3}}{2\left(m^{3}(m-2) a_{4}+u\left(m^{4}+2 m^{3}-4 m^{2}+16\right) a_{5}\right)\left((m-2)^{2} m^{3} a_{4}^{2}+2 m^{3} u\left(m^{2}-4\right) a_{4} a_{5}+u^{2}(m+2)^{2}\left(m^{3}-4 m+8\right) a_{5}^{2}\right)}$, $a_{2}=-m^{2}(3 m-2)\left(\frac{m}{m+2}\right)^{1-m} \frac{(m-2) a_{4}+(m+2) u a_{5}}{m^{3}(m-2) a_{4}+u\left(m^{4}+2 m^{3}-4 m^{2}+16\right) a_{5}}$,

$a_{3}=-(m-2) \frac{\left(m(m-2) a_{4}+u(m+2)^{2} a_{5}\right)^{3}}{2\left((m-2)^{2} m^{3} a_{4}^{2}+2 m^{3} u\left(m^{2}-4\right) a_{4} a_{5}+u^{2}(m+2)^{2}\left(m^{3}-4 m+8\right) a_{5}^{2}\right)}$,

where $u=\left(\frac{m}{m+2}\right)^{m}$.

In 2016, Behl et. al. [18], developed a four parametric based optimal fourth-order convergent iterative scheme as follows:

$$
\begin{aligned}
y_{n} & =x_{n}-\frac{2 m}{m+2} \frac{f\left(x_{n}\right)}{f^{\prime}\left(x_{n}\right)}, \\
x_{n+1} & =x_{n}-\frac{1}{2}\left[\frac{f\left(x_{n}\right)\left((5 m+2) b_{1} f^{\prime}\left(x_{n}\right)-(m+2) b_{2} f^{\prime}\left(y_{n}\right)\right)}{4 m\left(f^{\prime}\left(x_{n}\right)\right)^{2}}+\frac{2 m b_{3} f\left(x_{n}\right)}{(m-2) f^{\prime}\left(x_{n}\right) b_{4}+(m+2) f^{\prime}\left(y_{n}\right)}\right] .
\end{aligned}
$$

Here $b_{1}, b_{2}, b_{3}$ and $b_{4}$ are parameters, of which $b_{4}$ is a free parameter and others are given as

$$
\begin{aligned}
& b_{1}=-m^{2} \frac{(m-2)^{2} m^{3} a_{3}^{2}+3 v(m-2) m^{2}(m+2)^{2} a_{3}+2 v^{2}(m+2)^{2}\left(m^{3}+3 m^{2}+2 m-4\right)}{v^{2}(m+2)^{2}(5 m+2)}, \\
& b_{2}=-m^{5} \frac{(m-2) a_{3}+v(m+2)}{v^{2}(m+2)^{2}}, \\
& b_{3}=\frac{\left(\left(m^{2}-2 m\right) a_{3}+v(m+2)^{2}\right)^{3}}{8(v(m+2))^{2}}
\end{aligned}
$$

where $v=\left(\frac{m}{m+2}\right)^{m}$.

In 2016, Kim and Geum [20], presented a tri-parametric fourth order convergent family of optimal methods given by:

$$
\begin{aligned}
y_{n} & =x_{n}-\frac{2 m}{m+2} \frac{f\left(x_{n}\right)}{f^{\prime}\left(x_{n}\right)}, \\
x_{n+1} & =y_{n}-\left(\frac{a}{f^{\prime}\left(x_{n}\right)}+\frac{b}{f^{\prime}\left(y_{n}\right)}\right) f\left(x_{n}\right)-\left(\frac{c}{f^{\prime}\left(x_{n}\right)}+\frac{d}{f^{\prime}\left(y_{n}\right)}\right) F\left(y_{n}\right)
\end{aligned}
$$


where

$$
F\left(y_{n}\right)=f\left(x_{n}\right)+\left(y_{n}-x_{n}\right) \frac{\lambda f^{\prime}\left(x_{n}\right) f^{\prime}\left(y_{n}\right)}{f^{\prime}\left(x_{n}\right)+\rho f^{\prime}\left(y_{n}\right)},
$$

and $\lambda, \rho, d \in \mathbb{R}$ are free parameters. Similar bi-parametric schemes have also been suggested by Kim and Geum in [21,22].

More recently, Lee et al. in [19] have defined a tri-parametric two-point optimal fourth order method as follows:

$$
\begin{aligned}
y_{n} & =x_{n}-m \frac{f\left(x_{n}\right)}{f^{\prime}\left(x_{n}\right)+\lambda f\left(x_{n}\right)}, \\
x_{n+1} & =x_{n}-m W_{f}(s) \frac{f\left(x_{n}\right)}{f^{\prime}\left(x_{n}\right)+2 \lambda f\left(x_{n}\right)},
\end{aligned}
$$

where $s=\left(\frac{f\left(y_{n}\right)}{f\left(x_{n}\right)}\right)^{\frac{1}{m}}$ and

$$
W_{f}(s)=\frac{s\left(1+(c+2) s+r s^{2}\right)}{1+c s}
$$

being $\lambda, c, r \in \mathbb{R}$ free parameters.

In this paper, we are interested in presenting a two point family of optimal fourth order convergent parametric based iterative methods for multiple roots that can allow us to choose the class of stable members from the family of methods. Our method has a simple body structure involving two functions and a derivative evaluation and it is also capable of recapturing scheme (5) as a special case.

Because of this simple structure, there exist many subfamilies that can be defined, all of them with the same order of convergence, depending on one or more parameters. The selection of any of these iterative methods is a hard task provided if it is expected to be more stable or efficient than the rest. From the past decade, the tools of complex dynamics have made it possible. The qualitative properties of iterative schemes on the most simple nonlinear functions (quadratic polynomials) give feasible information about their behavior on more complicated functions. These have been clearly stated in many scientific works, as [23-26] where the asymptotic behavior of fixed and critical points with their respective convergence properties to the roots of the polynomial generates the sets of values of the involved parameter that give information about iterative methods with stable behavior and other related chaotic behavior and convergence to undesired elements, periodic orbits or fixed points that are not roots of the nonlinear equation.

The rest of the paper is in the following order: in Section 2, we define a new two-point fourth-order family of methods and analyze its convergence. Some special cases are given in Section 3. In Section 4, a dynamical analysis of the proposed class is made, in order to select those members of the family with better stability properties. Moreover these results are compared with those obtained by Lee et al. in [19]. In Section 5, a numerical comparison of the proposed methods with the existing ones is given. Conclusions are stated in Section 6.

\section{Construction of Optimal Fourth-Order Scheme}

Our primary aim is to develop an extended method to (5) by replacing the weight function $W_{f}(u)$ with a more general form $u H(u)$ to be discussed below.

Let $\xi$ be a multiple zero of $f: \mathbb{C} \rightarrow \mathbb{C}$ with integer multiplicity $m \geq 1$, and $f$ be an analytic function in the neighborhood of $\xi$. Then, for a given $x_{0}$, we give an iterative class in order to find an approximation of $\xi$ :

$$
\begin{aligned}
y_{n} & =x_{n}-m \frac{f\left(x_{n}\right)}{f^{\prime}\left(x_{n}\right)+a_{1} f\left(x_{n}\right)}, \\
x_{n+1} & =y_{n}-m u_{n} H\left(u_{n}\right) \frac{f\left(x_{n}\right)}{f^{\prime}\left(x_{n}\right)+a_{2} f\left(x_{n}\right)},
\end{aligned}
$$

where $a_{1}$ and $a_{2}$ are free parameters and the weight function $H: \mathbb{C} \rightarrow \mathbb{C}$ is analytic in the neighborhood of 0 , with $u_{n}=$ $\left[\frac{f\left(y_{n}\right)}{f\left(x_{n}\right)}\right]^{\frac{1}{m}}$.

We, now, investigate the convergence analysis of the proposed family (6) and find the necessary conditions on weight function $H\left(u_{n}\right)$ to assure the fourth-order convergence in the next result.

Theorem 1 Let $f: \mathbb{C} \rightarrow \mathbb{C}$ be an analytic function in the neighborhood of the multiple zero $\xi$ of $f$ with multiplicity $m \geq 1$. Additionally, let $H: \mathbb{C} \rightarrow \mathbb{C}$ is an analytic function in the neighborhood of 0 . Then, for a given $x_{0}$ in the neighborhood of $\xi$, the family of iteration functions (6) has fourth-order convergence with the following conditions:

$$
H(0)=1, H^{\prime}(0)=2, a_{2}=2 a_{1},
$$


and $\left|H^{\prime \prime}(0)\right|<\infty$.

Proof Let $\xi$ be a multiple zero of $f(x)$ and $e_{n}=x_{n}-\xi$ be the error at the $n t h$-iteration. Expanding $f\left(x_{n}\right)$ and $f^{\prime}\left(x_{n}\right)$ about $x=\xi$ by using the Taylor's series expansion, we have

$$
f\left(x_{n}\right)=\frac{f^{(m)}(\xi)}{m !} e_{n}^{m}\left[1+c_{1} e_{n}+c_{2} e_{n}^{2}+c_{3} e_{n}^{3}+c_{4} e_{n}^{4}+O\left(e_{n}^{5}\right)\right],
$$

and

$$
f^{\prime}\left(x_{n}\right)=\frac{f^{(m)}(\xi)}{m !} e_{n}^{m-1}\left[1+(m+1) c_{1} e_{n}+(m+2) c_{2} e_{n}^{2}+(m+3) c_{3} e_{n}^{3}+(m+4) c_{4} e_{n}^{4}+O\left(e_{n}^{5}\right)\right],
$$

where $c_{k}=\frac{m !}{(m+k) !} \frac{f^{(m+k)}(\xi)}{f^{(m)}(\xi)}, k=1,2,3, \ldots$

By using expressions (8) and (9), we have

$$
\frac{f\left(x_{n}\right)}{f^{\prime}\left(x_{n}\right)+a_{1} f\left(x_{n}\right)}=\frac{1}{m} e_{n}-\frac{\left(a_{1}+c_{1}\right)}{m^{2}} e_{n}^{2}+\frac{A_{1}}{m^{3}} e_{n}^{3}+\frac{A_{2}}{m^{4}} e_{n}^{4}+O\left(e_{n}^{5}\right),
$$

where $A_{1}=\left(a_{1}+c_{1}\right)^{2}+m c_{1}^{2}-2 m c_{2}$ and $A_{2}=-\left(a_{1}+c_{1}\right)^{3}-2 m a_{1} c_{1}^{2}-m(m+2) c_{1}^{3}+4 m a_{1} c_{2}+m(4+3 m) c_{1} c_{2}-3 m^{2} c_{3}$.

Thus, by using expression (10) in the first step leads us to:

$$
y_{n}-\xi=\frac{\left(a_{1}+c_{1}\right)}{m} e_{n}^{2}-\frac{A_{1}}{m^{2}} e_{n}^{3}-\frac{A_{2}}{m^{3}} e_{n}^{4}+O\left(e_{n}^{5}\right)
$$

Moreover,

$$
u_{n}=\frac{\left(a_{1}+c_{1}\right)}{m} e_{n}-\frac{\left(a_{1}^{2}+3 a_{1} c_{1}+(m+2) c_{1}^{2}-2 m c_{2}\right)}{m^{2}} e_{n}^{2}+\frac{B_{1}}{2 m^{3}} e_{n}^{3}+O\left(e_{n}^{4}\right),
$$

where $B_{1}=5 a_{1}(3+m) c_{1}^{2}+\left(7(m+1)+2 m^{2}\right) c_{1}^{3}+2 c_{1}\left(5 a_{1}^{2}-m(7+3 m) c_{2}\right)+2\left(a_{1}^{3}-5 m a_{1} c_{2}+3 m^{2} c_{3}\right)$.

Therefore,

$$
e_{n+1}=\frac{D_{1}}{m} e_{n}^{2}+\frac{D_{2}}{m^{2}} e_{n}^{3}+\frac{D_{3}}{m^{3}} e_{n}^{4}+O\left(e_{n}^{5}\right)
$$

where

$$
D_{1}=(-1+H(0))\left(a_{1}+c_{1}\right) \text {. }
$$

Letting $H(0)=1$, we have:

$$
D_{2}=-\left(a_{1}+c_{1}\right)\left(-a_{2}+a_{1} H^{\prime}(0)+\left(-2+H^{\prime}(0)\right) c_{1}\right),
$$

that clearly leads to $H^{\prime}(0)=2$ and $a_{2}=2 a_{1}$. Upon simplification:

$$
e_{n+1}=\frac{a_{1}^{2}\left(-4+H^{\prime \prime}(0)\right)+2 a_{1} c_{1}\left(-7+H^{\prime \prime}(0)\right)+\left(-9-m+H^{\prime \prime}(0)\right) c_{1}^{2}+2 m c_{2}}{-2 m^{3}} e_{n}^{4}+O\left(e_{n}^{5}\right) .
$$

This completes the proof.

Remark. The proposed family (6) has a free parameter $a_{1}$. It is also apparent that it can recapture (5) for a special case of the weight function $H\left(u_{n}\right)$. Thus, the proposed family of methods has the flexibility of choosing appropriate weight functions as a multiplier at the second step in order to obtain a stable class of methods for finding multiple roots of non-linear equations.

\section{Some special cases of our scheme}

From the conditions of Theorem 1, we can obtain several new subclasses from our family of methods by using different cases of $H\left(u_{n}\right)$ in the proposed scheme:

$$
\begin{aligned}
y_{n} & =x_{n}-m \frac{f\left(x_{n}\right)}{f^{\prime}\left(x_{n}\right)+a_{1} f\left(x_{n}\right)}, \\
x_{n+1} & =y_{n}-m u_{n} H\left(u_{n}\right) \frac{f\left(x_{n}\right)}{f^{\prime}\left(x_{n}\right)+2 a_{1} f\left(x_{n}\right)},
\end{aligned}
$$

satisfying

$$
H(0)=1, H^{\prime}(0)=2
$$

and $H^{\prime \prime}(0)$ bounded. 
Some particular cases of the proposed scheme are given as follows:

Case 1: Let us consider the polynomial weight function $H\left(u_{n}\right)=1+2 u_{n}+\frac{k}{2} u_{n}^{2}$ satisfying (7), where $k$ is a free parameter. Thus class (6) takes the form:

$$
\begin{aligned}
y_{n} & =x_{n}-m \frac{f\left(x_{n}\right)}{f^{\prime}\left(x_{n}\right)+a_{1} f\left(x_{n}\right)}, \\
x_{n+1} & =y_{n}-m u_{n}\left(1+2 u_{n}+\frac{k}{2} u_{n}^{2}\right) \frac{f\left(x_{n}\right)}{f^{\prime}\left(x_{n}\right)+2 a_{1} f\left(x_{n}\right)} .
\end{aligned}
$$

Note that this case is obtained from (5) by setting $c=0$ and $r=\frac{k}{2}$.

Case 2: Consider the rational weight function $H\left(u_{n}\right)=\frac{1+\beta u_{n}+\alpha u_{n}^{2}}{1+(\beta-2) u_{n}+\gamma u_{n}^{2}+\eta u_{n}^{3}}$ satisfying (7), where $\alpha, \beta, \gamma$ and $\eta$ are free parameters. Now, class (6) has the expression:

$$
\begin{aligned}
y_{n} & =x_{n}-m \frac{f\left(x_{n}\right)}{f^{\prime}\left(x_{n}\right)+a_{1} f\left(x_{n}\right)}, \\
x_{n+1} & =y_{n}-m u_{n} \frac{1+\beta u_{n}+\alpha u_{n}^{2}}{1+(\beta-2) u_{n}+\gamma u_{n}^{2}+\eta u_{n}^{3}} \frac{f\left(x_{n}\right)}{f^{\prime}\left(x_{n}\right)+2 a_{1} f\left(x_{n}\right)} .
\end{aligned}
$$

Note that setting $a_{1}=\lambda, \beta-2=c, \gamma=\eta=0, \alpha=r$ reduces to method (5).

Case 2A-B: For $\alpha=0, \gamma=1, \eta=0$, we have:

$$
\begin{aligned}
y_{n} & =x_{n}-m \frac{f\left(x_{n}\right)}{f^{\prime}\left(x_{n}\right)+a_{1} f\left(x_{n}\right)}, \\
x_{n+1} & =y_{n}-m u_{n} \frac{1+\beta u_{n}}{1+(\beta-2) u_{n}+u_{n}^{2}} \frac{f\left(x_{n}\right)}{f^{\prime}\left(x_{n}\right)+2 a_{1} f\left(x_{n}\right)},
\end{aligned}
$$

holding $a_{1}$ and $\beta$ as free parameters.

Case 2C-D: For $\alpha=0$ or $\alpha=1, \beta=2$ and $\gamma=1$, we consider:

$$
\begin{aligned}
y_{n} & =x_{n}-m \frac{f\left(x_{n}\right)}{f^{\prime}\left(x_{n}\right)+a_{1} f\left(x_{n}\right)}, \\
x_{n+1} & =y_{n}-m u_{n} \frac{1+2 u_{n}}{1+u_{n}^{2}+\eta u_{n}^{3}} \frac{f\left(x_{n}\right)}{f^{\prime}\left(x_{n}\right)+2 a_{1} f\left(x_{n}\right)},
\end{aligned}
$$

being $a_{1}$ and $\eta$ disposable parameters.

\section{Selecting the most appropriate weight function: divergent cases}

As it has been previously stated, the new class includes a known one from Lee et al. [19], when an specific rational function is used as the weight function $H\left(u_{n}\right)$. In what follows, we expose the reasons why selecting a second-degree polynomial (Case-1) or a different rational function (Case-2) can be better options, in terms of stability.

In order to arrange this analysis, we study the dynamical properties of the rational function resulting from the application of our proposed family on the nonlinear function $p(z)=(z-a)^{m}(z-b)^{m}$, with two multiple roots of multiplicity $m$. This is the most simple nonlinear function containing two $m$-multiple roots and, although the results cannot be directly extrapolated to any nonlinear function, several analysis on different nonlinear problems confirm, in the numerical section, these results.

Now, let us recall some dynamical concepts to increase the readability (see [27]). Let $R: \widehat{\mathbb{C}} \longrightarrow \widehat{\mathbb{C}}$ be a rational function self mapping on a Riemann sphere $\widehat{\mathbb{C}}$, then the orbit of a point $z_{0} \in \widehat{\mathbb{C}}$ is defined by

$$
\left\{z_{0}, R\left(z_{0}\right), R^{2}\left(z_{0}\right), \ldots, R^{n}\left(z_{0}\right), \ldots\right\}
$$

where $R^{k}$ denotes the $k$-th composition of the map $R$ with itself. We analyze the phase plane of the map $R$ by classifying the starting points from the asymptotic behavior of their orbits. The point $z_{0}$ is called a fixed point if $R\left(z_{0}\right)=z_{0}$ and it is a periodic point of period $p>1$ if $R^{p}\left(z_{0}\right)=z_{0}$ and $R^{k}\left(z_{0}\right) \neq z_{0}$, for $k<p$. A pre-periodic point is a point $z_{0}$ that is not periodic but there exists $k>0$ such that $R^{k}\left(z_{0}\right)$, is periodic.

Moreover, a fixed point of $R, z_{0}$, is called:

i) attracting if $\left|R^{\prime}\left(z_{0}\right)\right|<1$, or superattracting if $\left|R^{\prime}\left(z_{0}\right)\right|=0$,

ii) repulsive if $\left|R^{\prime}\left(z_{0}\right)\right|>1$ and 
iii) parabolic or neutral if $\left|R^{\prime}\left(z_{0}\right)\right|=1$.

Indeed, when $R$ depends also on one or several parameters, then $\left|R^{\prime}\left(z_{0}, \alpha_{1}, \ldots, \alpha_{k}\right)\right|$ is not an scalar, but a function of $\alpha_{i}$, for $i=1,2, \ldots, k$. It is called stability function of the fixed point and its value give us the character of the fixed point in terms of the value of $\alpha_{i}$, for $i=1,2, \ldots, k$.

Point $z_{0}$ is a critical point of $R$ if the derivative of the rational function at $z_{0}$ vanishes, that is $R^{\prime}\left(z_{0}\right)=0$. The asymptotic behavior of the critical points is a key fact for analyzing the stability of the method: a classical result (see, for example, [29]) sets that a critical point appears in each immediate basin of attraction, that is, in the connected component of the basin of attraction that holds the attracting element. By using this fact, and using these critical points as initial estimations, parameter planes are generated and their comprehension allows us to allocate all the unstable behavior depending on the parameters of the family, if they exist.

On the other hand, let us also remark that the fixed and critical points that are not the roots of the polynomial $p(z)$ are called strange fixed and free critical points, respectively.

The basin of attraction of an attractor $\xi$ is defined as:

$$
\mathcal{A}(\xi)=\left\{z_{0} \in \widehat{\mathbb{C}}: R^{n}\left(z_{0}\right) \rightarrow \xi, n \rightarrow \infty\right\}
$$

The Fatou set of the rational function $R$, is the set of points $z \in \widehat{\mathbb{C}}$ whose orbits tend to an attractor (fixed point, periodic point or infinity). Its complement in $\widehat{\mathbb{C}}$ is the Julia set, $\mathcal{J}(R)$. So the basin of attraction of any fixed point belongs to the Fatou set and the boundaries of these basin of attraction belong to the Julia set.

\subsection{Case 1: Polynomial weight function}

When the class (14) is applied on polynomial $p(z)$, a rational function $O_{p}(z)$ is obtained, depending on parameters $a_{1}, k$ and also on the multiple roots $a$ and $b$. To get a simpler operator, we use firstly the same conjugation as in Kim et al. paper [19], $a_{1}=\frac{k^{\prime}}{a-b}$ and also conjugacy maps (see the work of P. Blanchard in [27]) as Möbius transformation

$$
M(z)=\frac{z-a}{z-b}, \quad M^{-1}(z)=\frac{z b-a}{z-1}
$$

with properties:

$$
M(\infty)=1, \quad M(a)=0, \quad M(b)=\infty
$$

that yields a rational function that, being conjugated to $O_{p}(z)$ (and therefore, with equivalent dynamical behavior), does not longer depend on $a$ and $b$ :

$$
M_{p}\left(z, k^{\prime} k\right)=z^{4} \frac{\left(k^{\prime}-m z+m\right)^{2}}{\left(m(z-1)-k^{\prime} z\right)^{2}} \frac{N\left(z, k^{\prime}, k\right)}{D\left(z, k^{\prime}, k\right)},
$$

where $N\left(z, k^{\prime}, k\right)$ is the ninth-degree polynomial depending on parameters $k^{\prime}, k$ and also on the multiplicity $m$,

$$
\begin{aligned}
N\left(z, k^{\prime}, k\right)= & k^{\prime 4} m(z-1) z^{3}(k-2(9 z+8))+k^{3} m^{2}(z-1)^{2} z^{2}\left(8\left(4 z^{2}+8 z+3\right)-k(z+3)\right) \\
& -k^{\prime 2} m^{3}(z-1)^{3} z\left(4\left(7 z^{3}+23 z^{2}+21 z+4\right)-3 k(z+1)\right) \\
& +k^{\prime 4}(z-1)^{4}\left(-k(3 z+1)+12 z^{4}+56 z^{3}+88 z^{2}+48 z+4\right) \\
& -m^{5}(z-1)^{5}\left(2(z+1)^{2}\left(z^{2}+4 z+5\right)-k\right)+4 k^{\prime 5} z^{4}
\end{aligned}
$$

and $D\left(z, k^{\prime}, k\right)$ is the ninth-degree polynomial

$$
\begin{aligned}
D\left(z, k^{\prime}, k\right)= & k^{4} m z^{4}(z-1)((k-16) z-18)-k^{3} m^{2} z^{3}(z-1)^{2}\left(3(k-8) z^{2}+(k-64) z-32\right) \\
& +k^{\prime 2} m^{3} z^{2}(z-1)^{3}\left((3 k-16) z^{3}+3(k-28) z^{2}-92 z-28\right) \\
& -k^{\prime 4} z(z-1)^{4}\left((k-4) z^{4}+3(k-16) z^{3}-88 z^{2}-56 z-12\right) \\
& +m^{5}(z-1)^{5}\left((k-10) z^{4}-28 z^{3}-28 z^{2}-12 z-2\right)+4 k^{5} z^{5} .
\end{aligned}
$$

By solving equation $M_{p}\left(z, k^{\prime}, k\right)=z$, the fixed points of the rational function are obtained. Among them, $z=0$ and $z=\infty$, coming from the roots of the polynomial previous to the Möbius map. The asymptotic behavior of all the fixed points plays a key role in the stability of the iterative methods involved, as the convergence to fixed points different from the roots means an important drawback for an iterative method; so, we proceed now with this analysis. 
Remark. A direct result of the Möbius transformation applied on the rational function is the conjugacy by the inverse,

$$
\frac{1}{M_{p}\left(z, k^{\prime}, k\right)}=M_{p}\left(\frac{1}{z}, k^{\prime}, k\right) .
$$

Immediate consequences of this property are:

(a) If $z^{*}$ is a fixed point satisfying $M_{p}\left(z^{*}, k^{\prime}, k\right)=z^{*}$, then so it is $1 / z^{*}$, satisfying $M_{p}\left(1 / z^{*}, k^{\prime}, k\right)=1 / z^{*}$.

(b) $z=1$ is always an strange fixed point of $M_{p}\left(z, k^{\prime}, k\right)$, except maybe for some specific values of the parameters that simplify the operator.

Theorem 2 ([28], page 40) Let $\xi \in \mathbb{C}$ be a fixed point of a rational map $R$. Then, the multiplier $m(R, \xi)=R^{\prime}(\xi)$ is invariant under any conjugation $g$ (provided that the corresponding fixed point $g(\xi) \in \mathbb{C}$ ), that is, $m(R, \xi)=m\left(g \circ R \circ g^{-1}, g(\xi)\right)$.

By using this result, the following statement can be proven.

Corollary 1 Let $\xi \in \hat{\mathbb{C}}$ and $\bar{\xi}=\frac{1}{\xi} \in \hat{\mathbb{C}}$ be fixed points of the rational map $R$. Then both have the same character, as their stability functions coincide.

Proof The stability function of a fixed point of a rational function is, by definition, the absolute value of its multiplier. Let us define the conjugation $g: \hat{\mathbb{C}} \rightarrow \hat{\mathbb{C}}$, as $g(z)=\frac{1}{z}$. Then, in view of (18), we find that $M_{p}$ is conjugated to itself via conjugation $g(z)=\frac{1}{z}$. Hence, for $z \neq 0, \infty$, by applying Beardon's Theorem 2 we immediately obtain $M_{p}^{\prime}\left(\xi, k^{\prime}, k\right)=M_{p}^{\prime}\left(\bar{\xi}, k^{\prime}, k\right)$ after identifying $R=M_{p}\left(\xi, k^{\prime}, k\right)$.

For cases $z=0$ and $z=\infty$, we need to treat their multipliers separately to be described below. Let us observe that the conjugate fixed points $z=0$ and $z=\infty$ come from the roots of the polynomial $p(z)$. As they are roots of $M_{p}\left(z, k^{\prime}, k\right)=z$ of multiplicity four, it can be concluded that $M_{p}^{\prime}\left(0, k^{\prime}, k\right)=M_{p}^{\prime}\left(\infty, k^{\prime}, k\right)=0$. This is in concordance with the proven fourth-order of convergence of the proposed class of iterative methods.

As a direct consequence of this corollary, it can be stated that the conjugate fixed points of operator $M_{p}$ have the same stability, that is,

$$
\left|M_{p}^{\prime}\left(z^{*}, k^{\prime}, k\right)\right|=\left|M_{p}^{\prime}\left(1 / z^{*}, k^{\prime}, k\right)\right| .
$$

In order to study the stability of the strange fixed points of $M_{p}\left(z, k^{\prime}, k\right)$, we calculate its first derivative and evaluate it at every fixed point. Its stability function gives us information about the asymptotic behavior of the point. In our case, the stability of other fixed points depend on the value of parameters $k^{\prime}$ and $k$. We start this analysis with the strange fixed point coming from the divergence of the original method, $z=1$.

From this rational function, the following result can be stated.

Theorem 3 Let us consider the rational function $M_{p}\left(z, k^{\prime}, k\right)$. If $k^{\prime} \neq 0, z=1$ is a parabolic strange fixed point of $M_{p}$, with independence of the value of parameter $k$ as well as the multiplicity $m$. If $k^{\prime}=0$, then $z=1$ is attracting wether $|k-80|>256$, repulsive if $|k-80|<256$ and parabolic for $k$ such that $|k-80|=256$.

Proof From the nature of Möbius transformation, $z=1$ is a fixed point of the resulting rational function (except for specific values of the parameters that can make the rational function simpler). By definition, its stability is directly deduced from the derivative of the rational operator:

$$
M_{p}^{\prime}\left(z, k^{\prime}, k\right)=z^{3} \frac{2\left(m(z-1)-k^{\prime}\right)\left(k^{\prime} z-m z^{2}+m\right)^{5}}{\left(m(z-1)-k^{\prime} z\right)^{3}} \frac{\tilde{N}\left(z, k^{\prime}, k\right)}{\left(\tilde{D}\left(z, k^{\prime}, k\right)\right)^{2}}
$$

being $\tilde{N}\left(z, k^{\prime}, k\right)$ and $\tilde{D}\left(z, k^{\prime}, k\right)$ tenth- and ninth-degree polynomials, respectively. It can be easily checked that, for $k^{\prime} \neq 0$, $M_{p}^{\prime}\left(1, k^{\prime}, k\right)=1$ and this gives the neutral character of the fixed point, with independence of $k$ and $k^{\prime}$.

On the other hand, when $k^{\prime}=0$,

$$
M_{p}^{\prime}(z, 0, k)=-z^{3} \frac{4(z+1)^{6}\left(k\left(2 z^{2}-3 z+2\right)-20(z+1)^{2}\right)}{\left(-(k-10) z^{4}+28 z^{3}+28 z^{2}+12 z+2\right)^{2}}
$$

and then $M_{p}^{\prime}(1,0, k)=-\frac{256}{k-80}$ and the thesis of the theorem is straightforward. 
The importance of this result is related with the role of $z=1$ in Möbius map: as $M(\infty)=1, z=1$ in the conjugate operator corresponds to the divergence of the original one. So, an attracting or parabolic behavior of $z=1$ derives in divergent behavior of the iterative process, and it must be avoided. This is the reason why, in the following, we restrict ourselves to the case $k^{\prime}=0$ and analyze the stability of the resulting one-parametric rational function

$$
R(z, k) \equiv M_{p}(z, 0, k)=-z^{4} \frac{2(z+1)^{2}\left(z^{2}+4 z+5\right)-k}{(k-10) z^{4}-28 z^{3}-28 z^{2}-12 z-2} .
$$

According to Theorem 3, there exists an infinite set of iterative methods in the original family (14) whose behavior on $p(z)$ does not include the divergence. Our proposal has an advantage respect the family proposed by Lee et al. [19]: the rational operator $R(z, k)$ (and therefore, its dynamical behavior) does not depend on multiplicity $m$.

\subsection{Case 2: Rational weight functions}

Two general classes of rational weight functions have been described in the previous section, denoted as Cases 2A-B (see (20)) and 2C-D (see (17)). If a study is made on these subfamilies of methods, similar to the previous one with polynomial weight function, by applying them on $p(z)=(z-a)^{m}(z-b)^{m}$ and analyzing the conjugacy classes resulting from Möbius transformation, we get the following results.

In Case 2A-B, the resulting rational operator is

$$
M_{p}^{2}\left(z, k^{\prime}, \beta\right)=z^{4} \frac{\left(k^{\prime}-m z+m\right)^{2}}{\left(m(z-1)-k^{\prime} z\right)^{2}} \frac{N^{2}\left(z, k^{\prime}, \beta\right)}{D^{2}\left(z, k^{\prime}, \beta\right)},
$$

being

$$
\begin{aligned}
N^{2}\left(z, k^{\prime}, \beta\right)= & 2 \beta k^{\prime 4} z^{3}-k^{\prime 3} m(z-1) z^{2}(\beta(5 z+6)-1)+2 k^{\prime 2} m^{2}(z-1)^{2} z\left(3 \beta+(2 \beta+1) z^{2}+6 \beta z+z-1\right) \\
& -k^{\prime 3}(z-1)^{3}\left(2 \beta+(\beta+3) z^{3}+(7 \beta+6) z^{2}+(9 \beta+4) z-1\right) \\
& +m^{4}(z-1)^{4}(z+1)\left(2(\beta+1)+z^{2}+(\beta+2) z\right)
\end{aligned}
$$

and

$$
\begin{aligned}
D^{2}\left(z, k^{\prime}, \beta\right)= & 2 \beta k^{4} z^{4}-k^{\prime 3} m z^{3}(z-1)(5 \beta+(6 \beta-1) z)+2 k^{2} m^{2} z^{2}(z-1)^{2}\left(2 \beta+(3 \beta-1) z^{2}+6 \beta z+z+1\right) \\
& -k^{\prime 3} z(z-1)^{3}\left(\beta+(2 \beta-1) z^{3}+(9 \beta+4) z^{2}+(7 \beta+6) z+3\right) \\
& +m^{4}(z+1)(z-1)^{4}\left(2(\beta+1) z^{2}+(\beta+2) z+1\right)
\end{aligned}
$$

From this rational operator, it can be immediately deduced the following result, that gives us information about the presence of divergent behavior for any value of the disposable parameters $k$ and $\beta$ and for any value of the multiplicity $m$.

Theorem 4 Rational operator $M_{p}^{2}\left(z, k^{\prime}, \beta\right)$ has $z=1$ as an strange fixed point. Moreover, the character of $z=1$ is parabolic, with independence of the value of parameters $k^{\prime}$ and $\beta$, as well as the multiplicity $m$.

This parabolic character of $z=1$ yields us to the rejection of this specific case of rational functions, as the unstable behavior will be present for any value of the involved parameters.

In Case $2 \mathrm{C}-\mathrm{D}$, the rational function associated to the class acting on polynomial $p(z)$ is:

$$
M_{p}^{3}\left(z, k^{\prime}, \eta\right)=z^{4} \frac{\left(k^{\prime}-m z+m\right)^{2}}{\left(m(z-1)-k^{\prime} z\right)^{2}} \frac{N^{3}\left(z, k^{\prime}, \eta\right)}{D^{3}\left(z, k^{\prime}, \eta\right)},
$$

where

$$
\begin{aligned}
N^{3}\left(z, k^{\prime}, \eta\right)= & 2 k^{\prime 6} z^{5}(2+\eta)+m^{6}(-1+z)^{6}(1+z)\left(5+15 z^{2}+6 z^{3}+z^{4}+z(16+\eta)\right) \\
& -k^{\prime 5} m(-1+z) z^{4}(18+7 \eta+z(18+5 \eta)) \\
& -k^{\prime 5}(-1+z)^{5}\left(2+42 z^{4}+7 z^{5}+z(35+2 \eta)+z^{3}(97+3 \eta)+z^{2}(96+7 \eta)\right) \\
& -k^{\prime 3} m^{3}(-1+z)^{3} z^{2}\left(28+5 \eta+z^{3}(35+\eta)+7 z(17+3 \eta)+z^{2}(122+13 \eta)\right) \\
& +k^{\prime 2} m^{4}(-1+z)^{4} z\left(12+21 z^{4}+\eta+z^{3}(101+3 \eta)+z(93+11 \eta)+z^{2}(163+15 \eta)\right) \\
& \left.+k^{\prime 4} m^{2}(-1+z)^{2} z^{3}\left(32+9 \eta+z^{2}(34+4 \eta)+z(74+17 \eta)\right)\right)
\end{aligned}
$$


and

$$
\begin{aligned}
D^{3}\left(z, k^{\prime}, \eta\right)= & 2 k^{\prime 6} z^{6}(2+\eta)+m^{6}(-1+z)^{6}(1+z)\left(1+6 z+15 z^{2}+5 z^{4}+z^{3}(16+\eta)\right) \\
& -k^{\prime 5} m(-1+z) z^{5}(18+5 \eta+z(18+7 \eta)) \\
& -k^{\prime 5}(-1+z)^{5} z\left(7+42 z+2 z^{5}+z^{4}(35+2 \eta)+z^{2}(97+3 \eta)+z^{3}(96+7 \eta)\right) \\
& -k^{\prime 3} m^{3}(-1+z)^{3} z^{3}\left(35+\eta+7 z^{2}(17+3 \eta)+z^{3}(28+5 \eta)+z(122+13 \eta)\right) \\
& +k^{\prime 2} m^{4}(-1+z)^{4} z^{2}\left(21+z^{4}(12+\eta)+z(101+3 \eta)+z^{3}(93+11 \eta)+z^{2}(163+15 \eta)\right) \\
& \left.+k^{\prime 4} m^{2}(-1+z)^{2} z^{4}\left(34+4 \eta+z^{2}(32+9 \eta)+z(74+17 \eta)\right)\right)
\end{aligned}
$$

It can be also deduced the following result, where the unity is stated as strange fixed point of $M_{p}^{3}\left(z, k^{\prime}, \eta\right)$ and its character is proved, depending on parameters $k^{\prime}$ and $\eta$.

Theorem 5 Rational function $M_{p}^{3}\left(z, k^{\prime}, \eta\right)$ has $z=1$ as a parabolic strange fixed point, with independence of the value of parameter $\eta$ as well as the multiplicity $m$, if $k^{\prime} \neq 0$ and $\eta \neq-44$. If $k^{\prime}=0$ and $\eta \neq-44, z=1$ is an attracting strange fixed point if $|\eta+76|<16$, repulsive if $|\eta+76|>16$ and parabolic for $\eta$ such that $|\eta+76|=16$.

Proof The thesis is straightforward by checking the following aspects:

- $M_{p}^{3}\left(1, k^{\prime}, \eta\right)=1$ for all $k^{\prime} \in \mathbb{C}$ and $\eta \neq-44$.

- If $k^{\prime}=0$ and $\eta=-44$, then

$$
M_{p}^{3}\left(z, k^{\prime},-44\right)=z^{4} \frac{z^{3}+7 z^{2}+22 z-6}{6 z^{3}-22 z^{2}-7 z-1}
$$

and it can be easily checked that $z=1$ is not a fixed point of $M_{p}^{2^{\prime}}\left(z, k^{\prime}, \eta\right)$.

- For $\eta \neq-44$, the stability function of $z=1$ as fixed point of $M_{p}^{3}\left(z, k^{\prime}, \eta\right)$ is

$$
\left(M_{p}^{3}\right)^{\prime}\left(1, k^{\prime}, \eta\right)=2 \frac{\eta+68}{\eta+44} .
$$

So, $z=1$ is attracting if $\left|2 \frac{\eta+68}{\eta+44}\right|<1$, that is, considering $\eta=a+i b$, if

$$
\begin{aligned}
(a+68)^{2}+b^{2} & <\frac{1}{4}\left((a+44)^{2}+b^{2}\right) \\
a^{2}+b^{2}+152 a & <-5520 \\
(a+76)^{2}+b^{2} & <(16)^{2}
\end{aligned}
$$

Therefore, it is parabolic in the boundary of this disk and repulsive outside it.

As there exists a wide region in the parameter space where the divergence of the original class is not possible, we continue the analysis of the subclass $S(z, \eta)=M_{p}^{3}(z, 0, \eta)$ in the following section, along with the Case 1 of polynomial weight functions.

\section{Stability of strange fixed points}

The rest of strange fixed points of rational functions $R(z, k)$ and $S(z, \eta)$ and their respective stability (depending on parameters $k$, or $\eta$ ) plays also an important role in the general analysis of our class. In this section, we develop this study for polynomial weight functions as well as rational one.

\subsection{Case 1: polynomial weight function}

The fixed points of rational function $R(z, k)$ are the roots of equation $R(z, k)=z$. We focus our attention on those different from $z=0, z=\infty$ and $z=1$, as the stability of the later ones has been already analyzed. If $k \neq-176$, where the rational function is simplified, they are those satisfying $2+14 z+42 z^{2}+(60+k) z^{3}+42 z^{4}+14 z^{5}+2 z^{6}=0$. The symmetry of this polynomial yields to the following result. 
Proposition 1 If $k \neq-176$, the strange fixed points of $R(z, k)$ different from $z=1$ are

$$
z_{i}^{+}=\frac{z_{i}+\sqrt{z_{i}^{2}-4}}{2}, z_{i}^{-}=\frac{z_{i}-\sqrt{z_{i}^{2}-4}}{2}=\frac{1}{z_{i}^{+}}, i=1,2,3,
$$

being $z_{i}, i=1,2,3$ the roots of the third-degree polynomial $r(t)=2 t^{3}+14 t^{2}+36 t+32+k$. Moreover, four of them are repulsive for any value of $k$ and the two left are simultaneously attractive for values of $k$ inside the disk $|k+119.699|<$ 56.2995 , being superattractive at $k \approx-96.62$.

Proof If $k=-176$, the rational operator is simplified and reduced to

$$
R(z,-176)=z^{4} \frac{z^{4}+6 z^{3}+14 z^{2}+14 z+93}{93 z^{4}+14 z^{3}+14 z^{2}+6 z+1}
$$

whose four strange fixed points different from $z=1$ are the roots of $t^{4}+9 t^{3}+38 t^{2}+9 t+1=0$, that is, $\frac{1}{4}(-9 \pm 3 i \sqrt{7} \mp \sqrt{2-54 i \sqrt{7}})$. The value of the stability function at these points is (for all of them) $R^{\prime}\left(z_{i}^{ \pm},-176\right)=5.40904, i=1,2,3$, so they are repulsive for any value of $k$.

For $k \neq-176$, it has been previously stated that the strange fixed points are the roots of the polynomial $q(x)=2+14 x+$ $42 x^{2}+(60+k) x^{3}+42 x^{4}+14 x^{5}+2 x^{6}$. As it is symmetric of odd degree, it can be reduced to a half-degree polynomial by direct division by $x^{3}$ and the change of variables $t=x+\frac{1}{x}$. This yields to $r(t)$, whose roots, $z_{1}, z_{2}$ and $z_{3}$ can be calculated in an exact way. Therefore, their analytical expressions are obtained by undoing the change of variables.

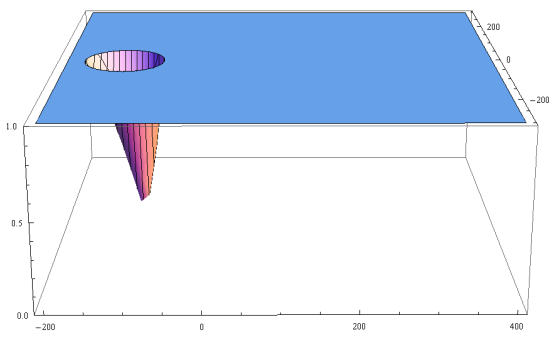

(a) $\left|R^{\prime}\left(z_{1}^{ \pm}\right), k\right|$

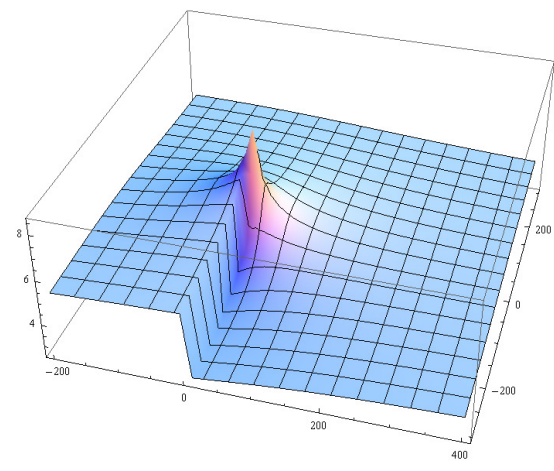

(b) $\left|R^{\prime}\left(z_{2}^{ \pm}\right), k\right|$

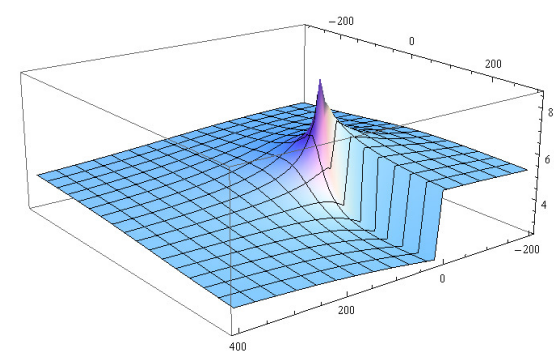

(c) $\left|R^{\prime}\left(z_{3}^{ \pm}\right), k\right|$

Fig. 1: Stability functions of strange fixed points $z_{i}^{ \pm}, i=1,2,3$

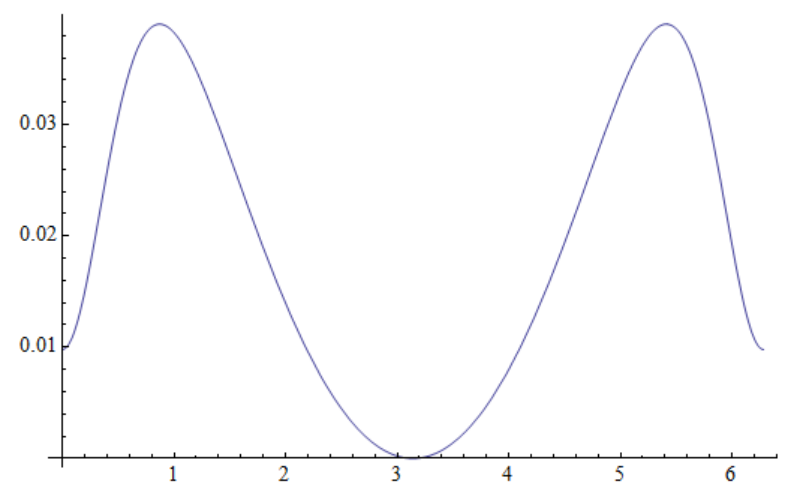

Fig. 2: $1-\left|R^{\prime}\left(z_{1}^{ \pm}, k\right)\right|$ for $k$ such that $|k+119.699|<56.2995$

The stability of the strange fixed points have been obtained numerically. In Figure 1 the stability function of each of the conjugate fixed points is presented, showing the repulsiveness of $x_{i}^{ \pm}, i=2,3$ and the loci of stability of $x_{1}^{ \pm}$(complex area 
where $x_{1}^{ \pm}$is attracting, that is, $\left|R^{\prime}\left(x_{1}^{ \pm}, k\right)\right|<1$. This loci of stability contains the disk $|k+119.699|<56.2995$, as has been numerically checked by calculating the error $1-\left|R^{\prime}\left(x_{1}^{ \pm}, k\right)\right|$ (see Figure 2).

By combining the results of Theorem 3 and Proposition 1, Figure 3 has been obtained. It shows that, excluding the stability area estimated by $|k+119.699|<56.2995$, the values of $k$ inside the complex disk $|k-80|<256$ make the rational operator $R(z, k)$ have only repulsive strange fixed points. Let us remark that this area is much bigger than that obtained in [19], for the

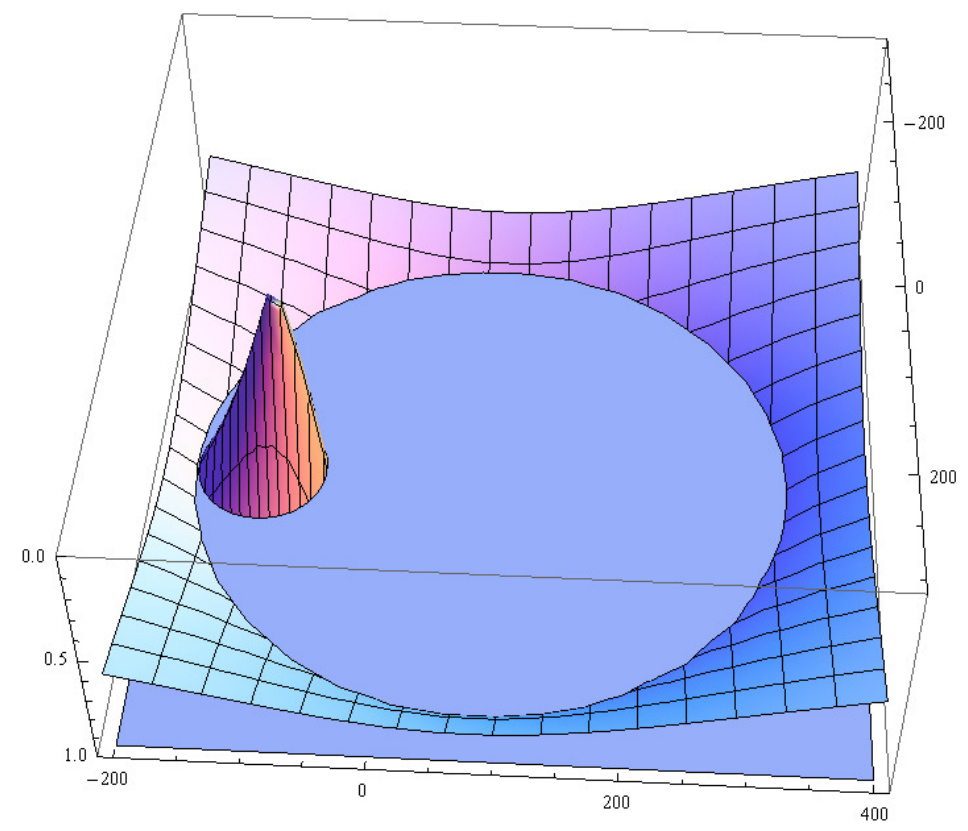

Fig. 3: Stability functions of attracting strange fixed points

operator resulting from a rational weight function. However, other kind of attracting undesired behavior can appear, such as periodic orbits.

\subsubsection{2-periodic orbits}

By analyzing the roots of the equation $R_{2}(z, k)=R(R(z, k), k)=z$, it is found that, besides the fixed points of $R(z, k)=0$, some of the 2 -periodic orbits are roots of $2+14 t+42 t^{2}+70 t^{3}+(80-k) t^{4}+70 t^{5}+42 t^{6}+14 t^{7}+2 t^{8}=0$ (the rest are roots of a 48th-degree polynomial). The stability function of the only pair of roots $t_{7,8}$ of this eighth-degree polynomial that is attracting can be seen at Figure 4a, where two areas of the complex plane, one of them close to the origin (whose detail can be seen at Figure 4b) and the second is a big disk including $|k-232.9|<102.5$. For values of $k$ inside these areas, there exist attracting periodic orbits of period two. It can be numerically checked that they are superattracting for $k \approx-7.22$ $\left(R_{2}^{\prime}\left(t_{7,8},-7.22\right) \approx 4.40 \cdot 10^{-5}\right)$ and $k \approx 221.1\left(R_{2}^{\prime}\left(t_{7,8}, 221.1\right) \approx 9.84 \cdot 10^{-10}\right)$. These stability regions, and also those corresponding to attracting strange fixed points, can be seen in Figure $4 \mathrm{c}$.

\subsection{Case 2: Rational weight function}

Let us consider now the case of rational operator $S(z, \eta)$. As the stability of strange fixed point $z=1$ has already been studied (see Theorem 5), and $z=0$ and $z=\infty$ are superattracting due to the fourth-order of convergence of the family (proven in Theorem 1), we focus ourselves in the calculation and analysis of the stability of the rest of strange fixed points.

Proposition 2 If $\eta \neq 0$, the strange fixed points of $S(z, \eta)$ different from $z=1$ are

$$
s_{i}^{+}=\frac{s_{i}+\sqrt{s_{i}^{2}-4}}{2}, s_{i}^{-}=\frac{s_{i}-\sqrt{s_{i}^{2}-4}}{2}=\frac{1}{s_{i}^{+}}, i=1,2,3,
$$

being $s_{i}, i=1,2,3$ the roots of the third-degree polynomial $r(t)=t^{3}+7 t^{2}+22 t+32+\eta$. Moreover, two of them, $s_{5}(\eta)$ and $s_{6}(\eta)$, are simultaneously attracting for a wide set of values of $\eta$ holding the region $|\eta+120|<27$, being superattracting at 


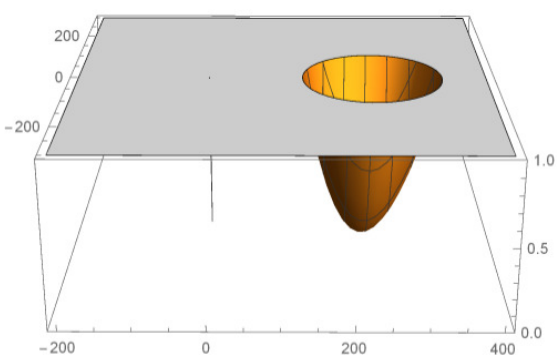

(a) Attracting 2-periodic orbits

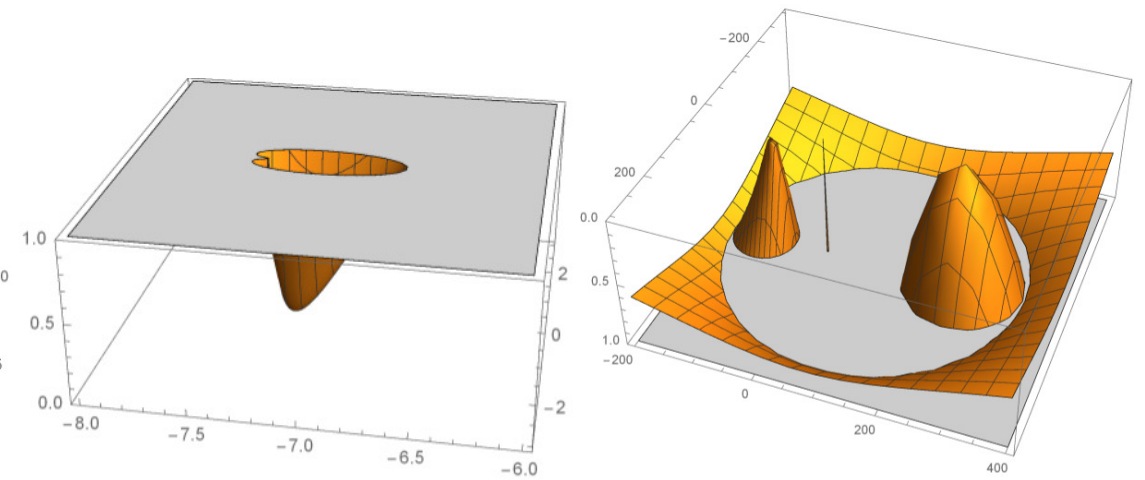

(b) A detail

(c) Complete set of stability functions

Fig. 4: Stability functions of periodic orbits

$\eta \approx-117.95$ and the four left are attracting (two-by-two) in two symmetric complex areas inside $[0.9,0.98] \pm i[1.62,1.72] ;$ they are superattracting for $\eta \approx 0.938074 \pm 1.66811 i$. When $\eta=0$, the strange fixed points of $S(z, 0)$ are $z=1$ and the four roots of polynomial $1+5 t+11 t^{2}+5 t^{3}+t^{4}$, that are simultaneously repulsive.

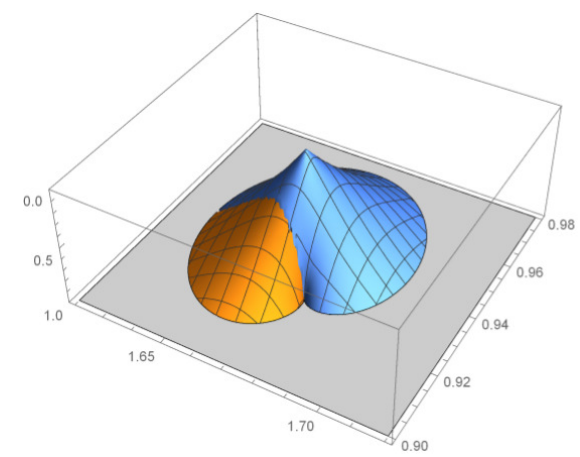

(a) $\left|S^{\prime}\left(s_{1,2}^{ \pm}\right), \eta\right|$ (orange) and $\left|S^{\prime}\left(s_{3,4}^{ \pm}\right), \eta\right|$ (blue)

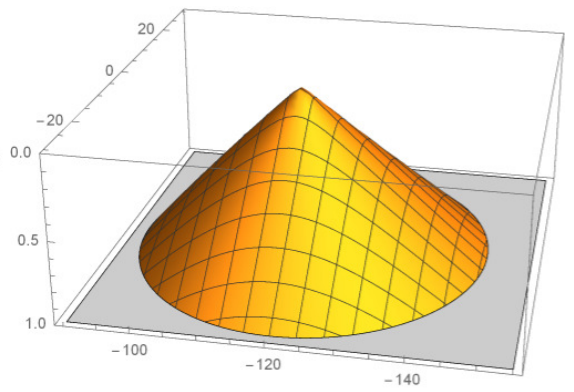

(b) $\left|S^{\prime}\left(s_{5,6}^{ \pm}\right), \eta\right|$

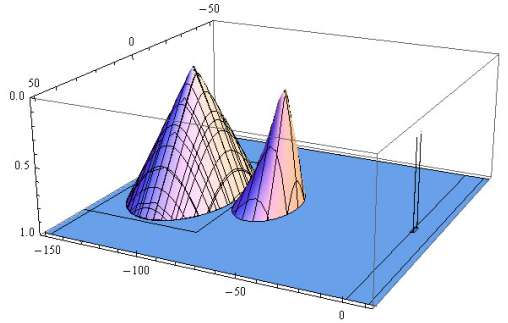

(c) Stability set

Fig. 5: Stability functions $\left|S^{\prime}\left(s_{i}^{ \pm}\right), \eta\right|$ of strange fixed points $s_{i}^{ \pm}, i=1,2,3$ and $z=1$

The proof of this result, being numerical, is similar to those made in the previous subsection, by analyzing the value of the stability function of each one of the conjugate strange fixed points. In Figure 5, these stability functions are presented and it can be observed (see Figure 5b) how at the right of the disk of stability of $z=1$ a bigger loci of stability of two strange fixed points appear (Figure 5a) including $|\eta+120|<27$, and also two smaller areas near the origin, in [0.9, 0.98] $\pm i[1.62,1.72]$, see Figure $5 \mathrm{~b}$ corresponding to two pairs of conjugated fixed points $\left(\left|S^{\prime}\left(s_{1,2}^{ \pm}\right), \eta\right|\right.$ appears in orange color and $\left|S^{\prime}\left(s_{3,4}^{ \pm}\right), \eta\right|$ in blue one). The union of all these stability functions, denoted by stability set, is presented in Figure $5 \mathrm{c}$.

By combining the results of Theorem 5 and Proposition 2, it is observed that, excluding the stability area of strange fixed points, there are wide areas of the complex plane (for example, values of $\eta$ with positive real part greater than 1 ) in which all there are no other attracting fixed point than the roots. It is possible that in this region attracting periodic orbits of different periods appear but, due to the complexity of the operator $S(S(z, \eta), \eta)$, they have not been calculated. However, they will appear in the parameter plane, where all the attracting elements different to the roots are visualized. It will be calculated in the following section.

\section{Critical points: parameter and dynamical planes}

A classical result establishes that there is at least one critical point associated with each invariant Fatou component. This is the main reason to calculate the free critical points of the rational function associated to the family of iterative methods (those critical points different to $z=\infty$ and $z=0$ ). All the attracting behavior are in the basins of attraction where these critical 
points lay. Thus, dangerous behavior, as convergence to undesired attracting fixed points or periodic orbits, of some members of the class of iterative methods can be located by analyzing these free critical points.

\subsection{Case 1: Polynomial weight function}

The following result is devoted to this aim, setting the number and analytical expressions of the critical points of $R(z, k)$

Theorem 6 The set of critical points of rational operator $R(z, k)$ is defined by $z=0, z=\infty$ and the free critical points. These are:

a) $z=-1$, that is a pre-periodic point, as $R(-1, k)=1$ for any $k \in \mathbb{C}$.

b) For $k \notin\{0,10,80\}$, there exist two free conjugate critical points,

$$
c r_{1}(k)=\frac{3 k-\sqrt{7} \sqrt{(80-k) k}+40}{4(k-10)}, c r_{2}(k)=\frac{3 k+\sqrt{7} \sqrt{(80-k) k}+40}{4(k-10)}=\frac{1}{c r_{1}(k)} .
$$

Proof By definition, the critical points are the roots of $R^{\prime}(z, k)=0$, that is, of

$$
-4 z^{3}(1+z)^{6}\left((2 k-20) z^{2}-(3 k+40) z+2 k-20\right)=0,
$$

so the free critical points are $z=-1, z=c r_{1}(k)$ and $z=c r_{2}(k)$. As the later two are not defined for $k=10$, we analyze the rational operator and see that it is simplified for this value of the parameter,

$$
R(z, 10)=z^{5} \frac{z^{3}+6 z^{2}+14 z+14}{14 z^{3}+14 z^{2}+6 z+1} .
$$

From this expression, it can be deduced that the iterative method corresponding to $k=10$ has fifth-order of convergence (on quadratic polynomials) and also that its only critical points are $z=0, z=\infty$ and $z=-1$. It can be also observed that $c r_{1}(k)=c r_{2}(k)$ for $k=0$ and $k=80$, but, by studying $R(z, k)$ at these two values, we found that it is also simplified

$$
\begin{aligned}
R(z, 0) & =z^{4} \frac{z^{2}+4 z+5}{5 z^{2}+4 z+1}, \\
R(z, 80) & =-z^{4} \frac{z^{3}+7 z^{2}+21 z+35}{35 z^{3}+21 z^{2}+7 z+1},
\end{aligned}
$$

in such a way that the only critical points are again (in both cases) $z=0, z=\infty$ and $z=-1$.

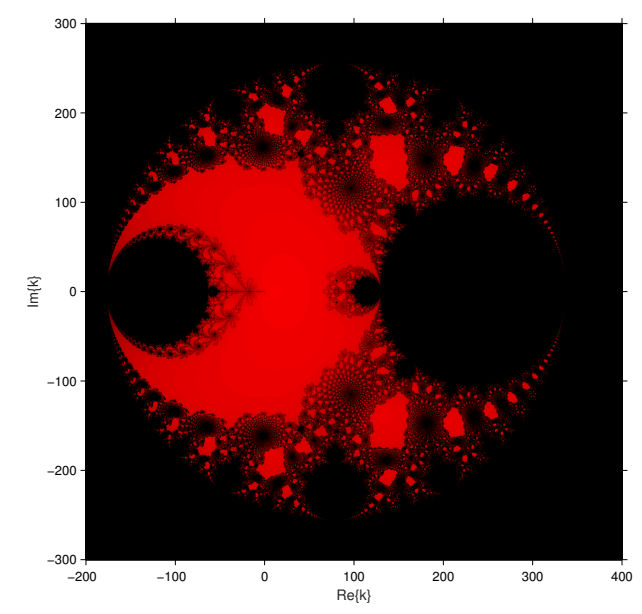

(a) Phase space

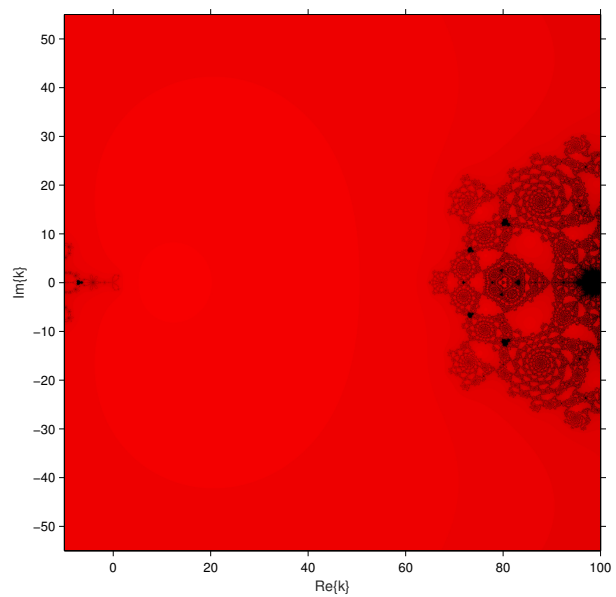

(b) A detail

Fig. 6: Parameter plane of $R(z, k)$

Points $z=\infty$ and $z=0$ are both superattractive fixed points of $R(z, k)$, so they also are critical points and give rise to their respective Fatou components [30]. Therefore, the way to calculate the parameter planes associate to $R(z, k)$ is to study 
the orbits of each free independent critical point for all the complex values of the parameter $k$ in the defined mesh. In this subsection, we analyze the only parameter plane of $R(z, k)$, as it has only one free independent critical point, $c r_{1}(k)$.

The way to calculate the parameter plane associate to $R(z, k)$ is the study of the orbits of the free independent critical point for all the complex values of the parameter $k$ in a defined mesh of 2000 points per axis. In order to get this parameter plane we study the orbits of the free critical point for each $k$ in the complex square $[-200,400] \times[-300,300]$, that includes all the stability functions of the fixed and periodic points analyzed in the previous section. By using the routines appearing in [31], we have painted in red the values of the parameter that makes the critical point converge to $z=0$ or $z=\infty$ (brighter as lower is the number of iterations needed), and in black if the critical point has not converged after 500 iterations, or has converged to another element (attracting strange fixed point or periodic orbit). We show the resulting parameter plane in Figure 6, including a detail of the central red area of stable behavior.

It is clear, by observing Figure 6, that all the structures of the stability functions of fixed and 2-periodic points plotted in Figure 4c (and analyzed in the results of the previous section) are identified. The rest of black structures correspond to attracting periodic orbits of higher periods. Also, the red area between the two biggest black areas corresponding to attracting strange fixed points (see Proposition 1) and attracting 2-periodic orbits (estimated by $|k-232.9|<102.5$ ) includes the real interval $[0,65]$ and it is much wider than that obtained in [19] for the rational weight function. All these real an complex values of parameter $k$ correspond to iterative methods of the proposed class whose only possible behavior is to converge to the roots. They are the best candidates to have stable behavior for any other kind of nonlinear functions.

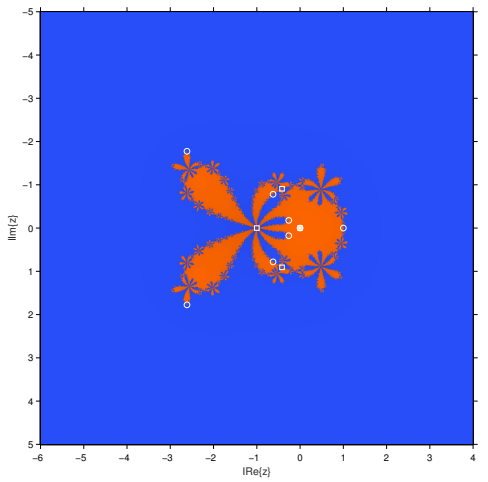

(a) $k=-5$

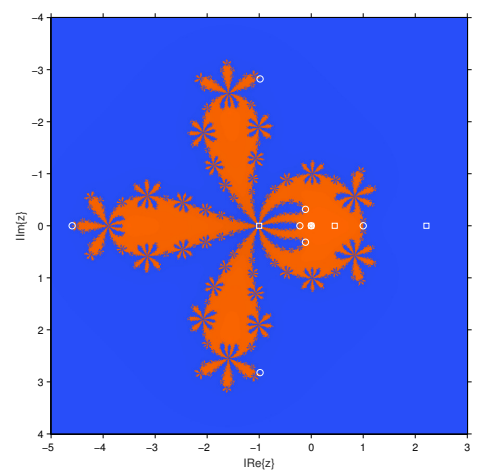

(d) $k=40$

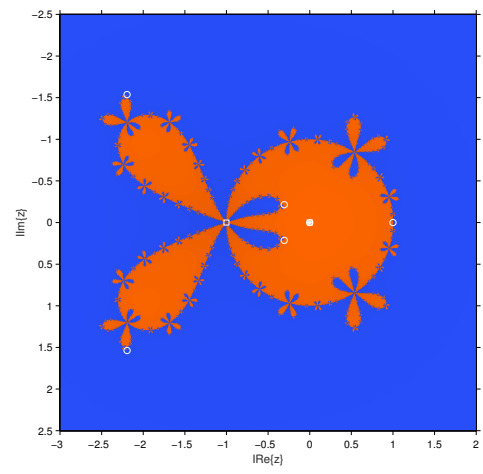

(b) $k=0$

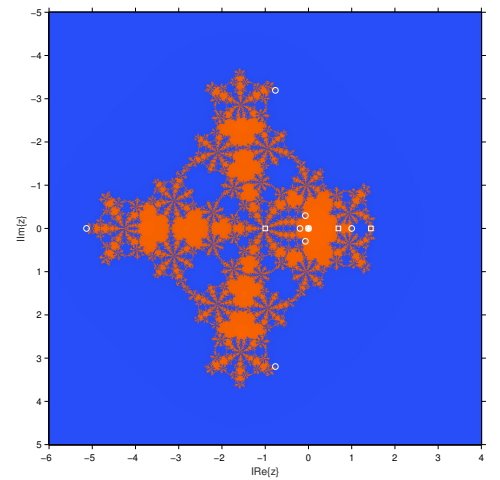

(e) $k=65$

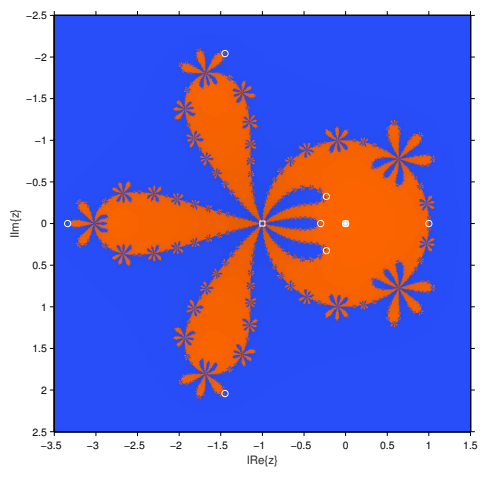

(c) $k=10$

Fig. 7: Dynamical planes corresponding to stable performance

This stable performance can be observed in the different cases presented in Figure 7, where the only basins of attraction are those corresponding to the original roots of the polynomial, that is, the basin of $z=0$ and that of $z=\infty$. These dynamical planes have been obtained by using the routines in [31], by means of a mesh of $800 \times 800$ points as initial guesses of the iterative method corresponding to an specific value of parameter $k$. Each one of this initial estimations is plotted in orange (blue) color if the method converges to zero (infinity) in less than 200 iterations or in black in other cases. Moreover, fixed points are marked with white circles, meanwhile attraction ones appear as white stars and critical points as white squares. In this way, it can be visualized as attracting fixed points lay in their own basin of attraction, that contains at least one critical 
point; on the other hand, repulsive fixed point always appear in the boundary of the basins of attraction, that is, in the Julia set.

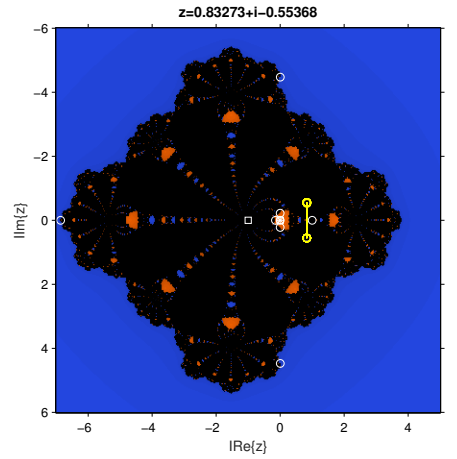

(a) $k=221$

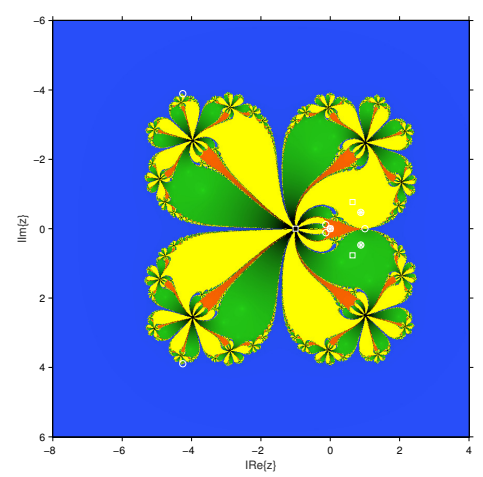

(d) $k=-150$

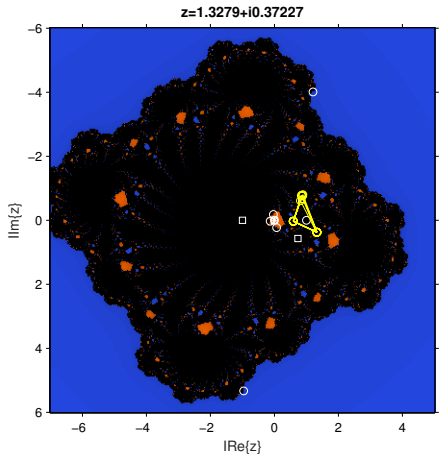

(b) $k=205+i 200$

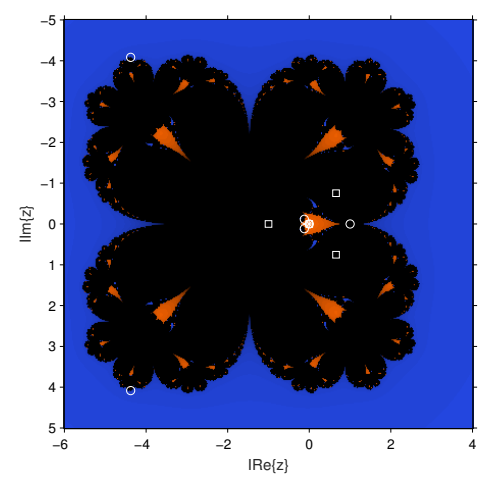

(e) $k=-176$

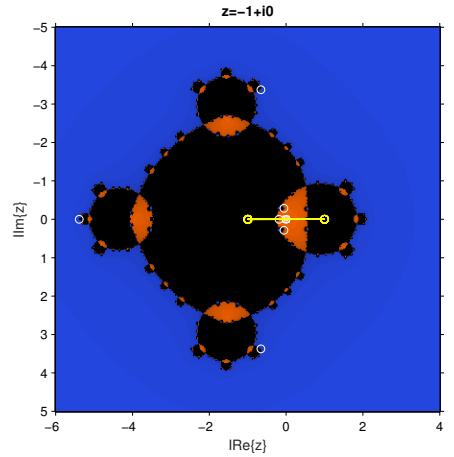

(c) $k=80$

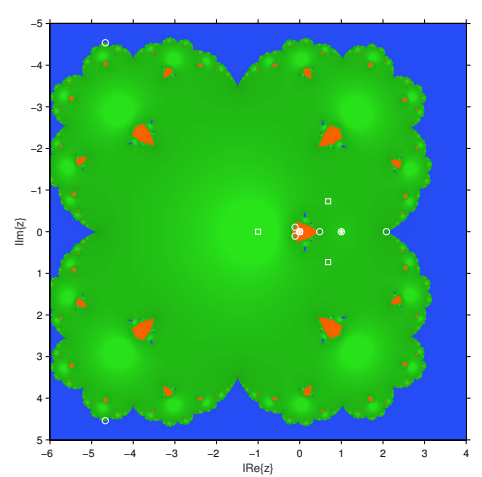

(f) $k=-250$

Fig. 8: Dynamical planes corresponding to unstable performance

The biggest area of stability of 2-periodic orbits is represented by a dynamical plane for $k=221$, very close to the value of $k$ where this orbit is superatracting. The black area in the dynamical plane (see Figure 8a) corresponds to the basin of attraction of the periodic orbit, that is plotted in yellow.

It is known, by Sharkowski's Theorem (see, for example, [29]), that the existence of 3-periodic orbits yields to the existence of periodic orbits of any period. In Figure 8b, one attracting 3-periodic orbit appears in the dynamical plane corresponding to $k=205+200 i$.

Although we have checked that $k=80$ yields to a simplified rational function with no other free critical point than $z=-1$, it does not appear in the set of stable behavior (Figure 7). The reason is that, for this value of parameter $k, z=1$ is no longer an strange fixed point and it can be proven that, not also $z=-1$ is a pre-image of $z=1$, but also $z=-1$ is an image of $z=1$; that is, both points form an attracting 2-periodic orbit. This can be visualized in Figure 8c, where yellow lines represent the orbit.

A different unstable behavior can be observed in Figure 8d, corresponding to $k=-150$, in the loci of stability of two attracting strange fixed points. For this value of $k$, these points are $0.8818 \pm 0.4717 i$ and their respective basins of attraction appear in yellow and green in the figure.

Another value of $k$ where the rational function $R(z, k)$ is simplified is $k=-176$; in it, four of the strange fixed points collapse in $z=1$. However, this does not means that it is more stable as, for this value of $k, z=1$ is parabolic and, therefore, it behaves as attracting or repulsing fixed point in different areas of the dynamical plane; corresponding to its stable manifold, it has a wide basin of attraction, as can be seen at Figure 8e. However, for $k=-250, z=1$ is attracting and its basin of attraction can be seen plotted in green color in Figure 8f. 


\subsection{Case 2: Rational weight function}

In case of fixed point operator $S(z, \eta)$, the critical points that will lead us through the parameter plane to find all the pathological behavior, are described in the following result.

Theorem 7 The set of critical points of rational operator $S(z, \eta)$ is defined by $z=0, z=\infty$ and (if $\eta \neq 0)$ eight free critical points, two-by-two dependent by conjugation. These are:

$$
c s_{i}^{+}(\eta)=\frac{1}{2}\left(u_{i}(\eta)+\sqrt{u_{i}(\eta)^{2}-4}\right), c s_{i}^{-}(\eta)=\frac{1}{2}\left(u_{i}(\eta)-\sqrt{u_{i}(\eta)^{2}-4}\right), \quad i=1,2,3,4,
$$

being $u_{i}(\eta), i=1,2,3,4$ the roots of polynomial $24 u^{4}+(188+5 \eta) u^{3}+(654+24 \eta) u^{2}+(1308+51 \eta) u+\left(1636+64 \eta+2 \eta^{2}\right)$. However, if $\eta=0$, there are only four critical points, roots of the polynomial $12+46 t+71 t^{2}+46 t^{3}+12 t^{4}$, as the four left collapse in $z=-1$, pre-image of $z=1$.

Proof By definition, critical points are found by solving the equation $S^{\prime}(z, \eta)=0$; in this case, it is equivalent to calculate the roots of $24+(188+5 \eta) t+(654+24 \eta) t^{2}+(1308+51 \eta) t^{3}+\left(1636+64 \eta+2 \eta^{2}\right) t^{4}+(1308+51 \eta) t^{5}+(654+24 \eta) t^{6}+(188+$ $5 \eta) t^{7}+24 t^{8}$. Due to the symmetry of this polynomial, its simple division of the polynomial by $t^{4}$ and by means of the change of variables $u=t+\frac{1}{t}$, the polynomial results as $24 u^{4}+(188+5 \eta) u^{3}+(654+24 \eta) u^{2}+(1308+51 \eta) u+\left(1636+64 \eta+2 \eta^{2}\right)$ (that can be analytically solved) and the original critical points can be found through its roots as it is stated in the thesis of this result. If $\eta=0$, the fixed point operator

$$
S(z, 0)=\frac{x^{4}\left(z^{2}+4 z+6\right)}{6 z^{2}+4 z+1}
$$

is simplified and there are only four critical points, conjugated two-by-two, obtained by solving $S^{\prime}(z, 0)=0$.

By using the same routines than in the case of polynomial weight function and stating a mesh of $800 \times 800$ points and a maximum of 200 iterations, the parameter planes associated to $S(z, \eta)$ are found and can be seen in Figure 9. In it, four parameter spaces are shown, corresponding to each one of the free independent critical points of $S(z, \eta)$; in this case, $c s_{i}^{+}(\eta)$, $i=1,2,3,4$, have been used as initial estimation in the corresponding parameter plane. As before, red areas correspond to values of parameter $\eta$ such that $S\left(c s_{i}^{+}(\eta), \eta\right)$ converges to $z=0$ or $z=\infty$, coming from the original roots of the polynomial. The values of $\eta$ that assure convergence only to the roots are those that are found to be simultaneously red in all the parameter planes. As can be observed, this area is wide enough to consider this rational weight function a stable selection.

From these results, several stable elements can be selected from the rational class of iterative methods: those corresponding to values of parameter $\eta$ where there no exist attracting strange fixed nor periodic points. The dynamical planes corresponding to some of them can be seen at Figure 10, where a mesh of $800 \times 800$ points have been used, with a maximum of 200 iterations. In Figure 10a, the case of $\eta$ null is presented, whose main characteristic is that the basins of the roots are disconnected. The same happens for Figure 10b where some black points appear simulating to connect the different components of the basins of attraction, but thy are only points of slower convergence. Again disconnected are the basins for Figure 10c. The best behavior appears for $\eta=-44$ (see Figure 10d), where the behavior is as that of Newton's method for simple roots (that is, global convergence) but with fourth-order of convergence. For this value of the parameter, the rational operator $S(z, \eta)$ is simplified and $z=1$ is not a fixed point.

In a similar way, any of the values of $\eta$ that appears in black in any of the parameter planes in Figure 9 can be selected as a unstable member of the rational class of iterative methods. In Figure 11, some of them are presented, as in Figure 11b, where the basins of simultaneous superattracting fixed points $-0.3870+0.2135 i$ and $-1.9811-1.0927 i$ corresponding to $\eta=$ $0.938074+1.66811 i$ appear. Also in Figure 11c, strange fixed points $z=0.5241$ and $z=1.9080$ are simultaneously attracting and show their own basin of attraction. A 2-periodic orbit appears in the dynamical plane of Figure 11d, corresponding to $\eta=-150$.

\section{Numerical results}

In this section, we investigate the numerical performance of our proposed fourth order schemes namely (19)-(20) and (21)-(24) denoted by S1A, S1B, S2A, S2B, S2C and S2D, respectively, by carrying out some standard non-linear test functions. From the stability analysis made in the previous section, the selected values of the parameters in each case give us expectations of good performance in cases $1 \mathrm{~A}, 2 \mathrm{C}$ and $2 \mathrm{D}$, meanwhile worse behavior can be expected in cases 1B, 2A, and 2B.

Case 1A: For $a_{1}=0, k=11$, we have:

$$
\begin{aligned}
y_{n} & =x_{n}-m \frac{f\left(x_{n}\right)}{f^{\prime}\left(x_{n}\right)}, \\
x_{n+1} & =y_{n}-m u_{n}\left(1+2 u_{n}+\frac{11}{2} u_{n}^{2}\right) \frac{f\left(x_{n}\right)}{f^{\prime}\left(x_{n}\right)} .
\end{aligned}
$$




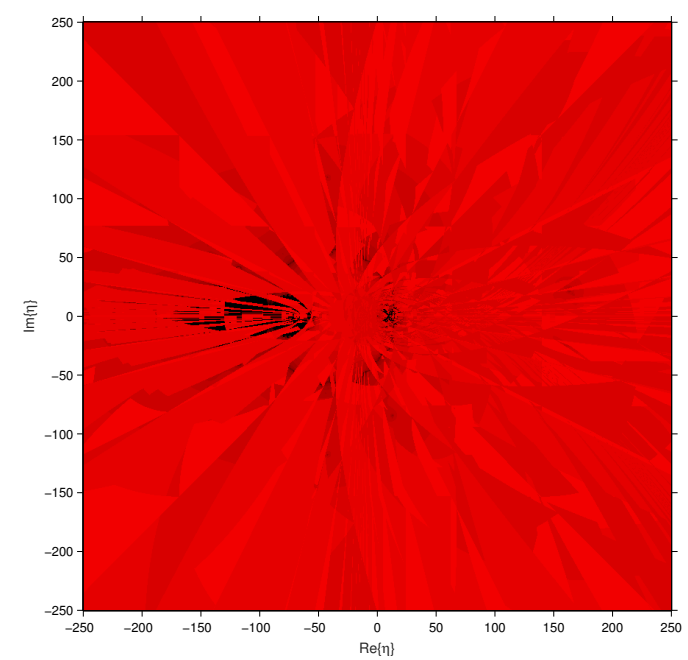

(a) $S\left(c s_{1}^{+}(\eta), \eta\right)$

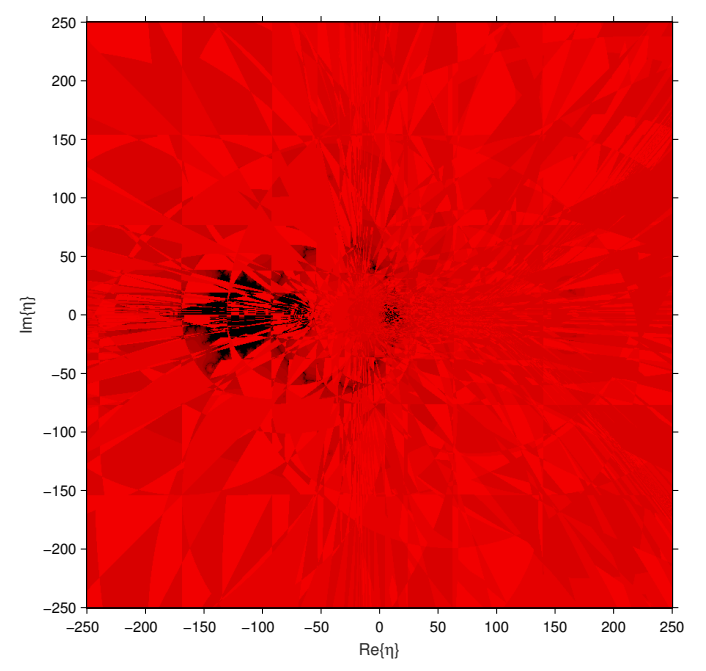

(c) $S\left(c s_{3}^{+}(\eta), \eta\right)$

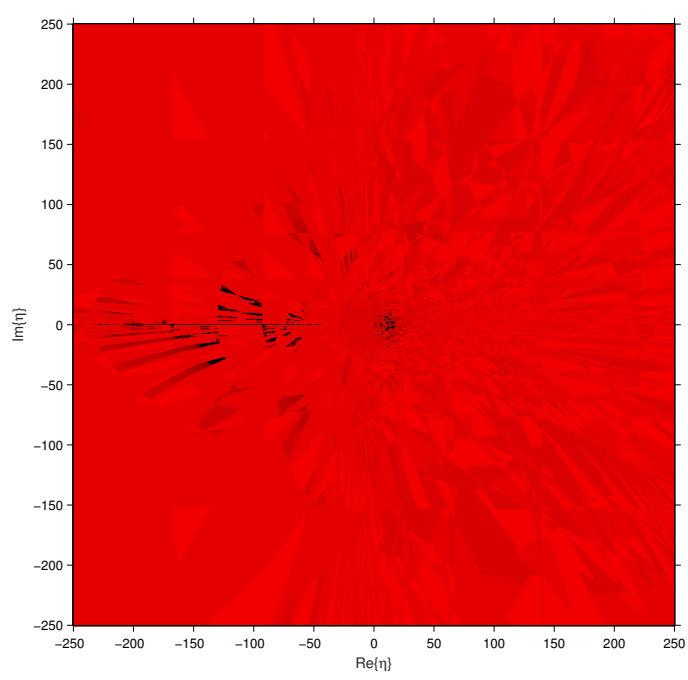

(b) $S\left(c s_{2}^{+}(\eta), \eta\right)$

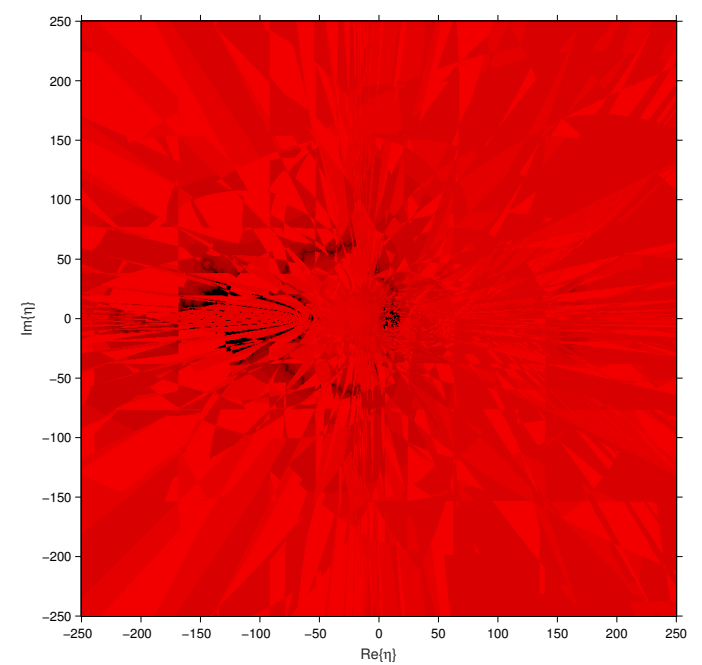

(d) $S\left(c s_{4}^{+}(\eta), \eta\right)$

Fig. 9: Parameter planes of $S(z, \eta)$

Let us remark that $a_{1}=0$ involves $k^{\prime}=0$ in the dynamical analysis (Theorem 3, Section 4.1) and, in this case, $k=11$ makes repulsive the strange fixed point $z=1$, coming from the divergence of the original method. So, divergence is avoided in this case. Moreover, Proposition 1 (Section 5) tell us that for this value of $k$, the strange fixed points are all repulsive. Regarding critical points, Theorem 6 in Section 6 assures us that $k=11$ is in the stable region of the parameter plane (see Figure 6).

Case 1B: For $a_{1}=\frac{m}{2}, k=11$, we have:

$$
\begin{aligned}
y_{n} & =x_{n}-m \frac{2 f\left(x_{n}\right)}{2 f^{\prime}\left(x_{n}\right)+m f\left(x_{n}\right)}, \\
x_{n+1} & =y_{n}-m u_{n}\left(1+2 u_{n}+\frac{11}{2} u_{n}^{2}\right) \frac{f\left(x_{n}\right)}{f^{\prime}\left(x_{n}\right)+m f\left(x_{n}\right)} .
\end{aligned}
$$

From Theorem 3, it can be deduced in this case that $z=1$ is parabolic; so, it can behave both as an attractor or as a repulsor. In this case, we can not assure that divergence is avoided. 


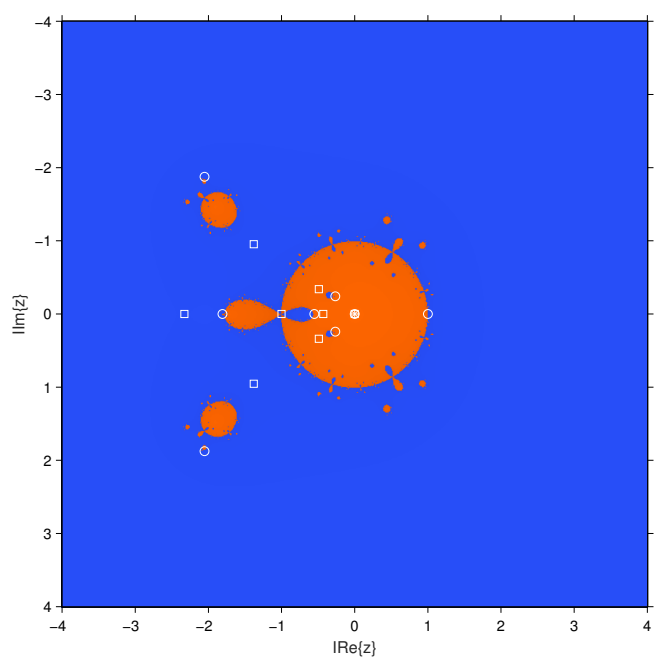

(a) $\eta=0$

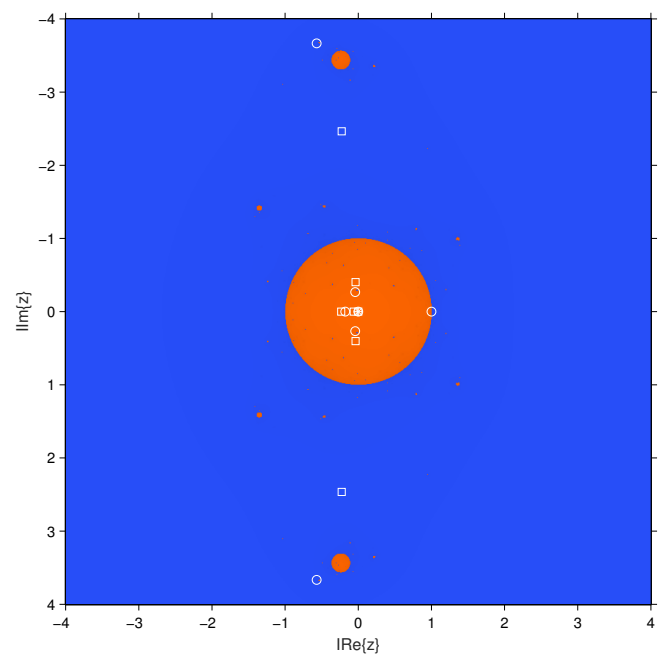

(c) $\eta=50$

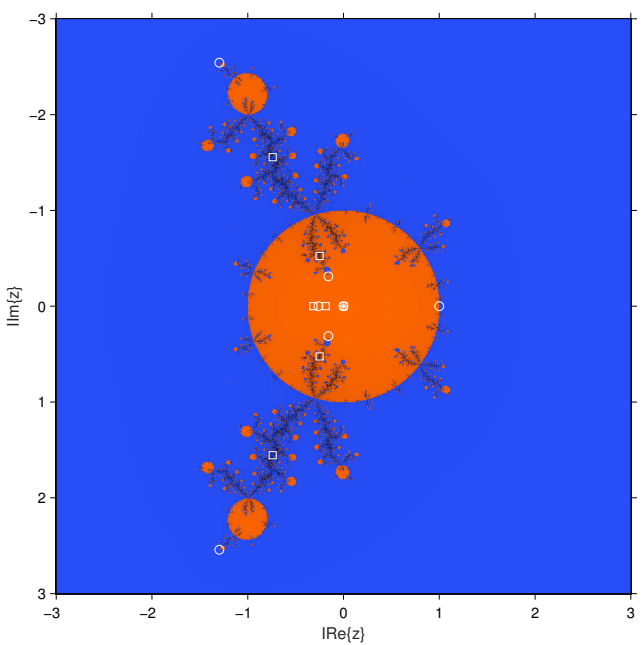

(b) $\eta=10$

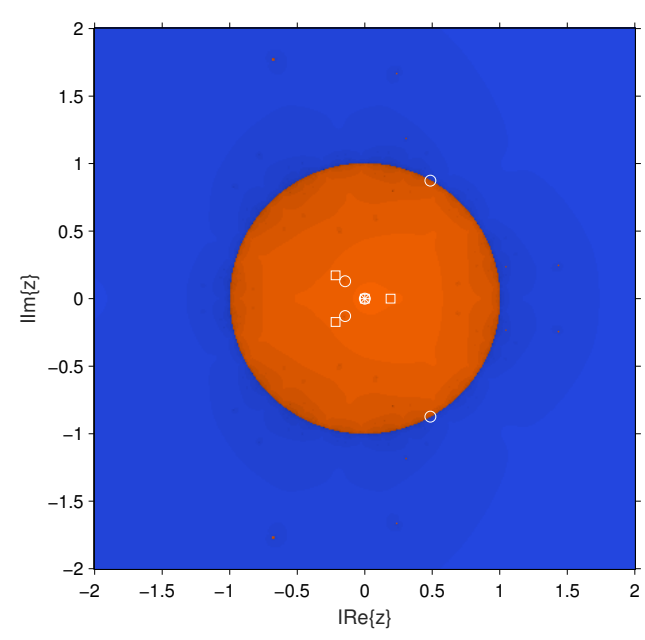

(d) $\eta=-44$

Fig. 10: Dynamical planes corresponding to stable performance of $s(z, \eta)$

Case 2A: For $a_{1}=0, \alpha=0, \beta=4, \gamma=1, \eta=0$, we have:

$$
\begin{aligned}
y_{n} & =x_{n}-m \frac{f\left(x_{n}\right)}{f^{\prime}\left(x_{n}\right)}, \\
x_{n+1} & =y_{n}-m u_{n} \frac{1+4 u_{n}}{\left(1+u_{n}\right)^{2}} \frac{f\left(x_{n}\right)}{f^{\prime}\left(x_{n}\right)} .
\end{aligned}
$$

This particular rational function yields to a parabolic strange fixed point $z=1$, so it will be unstable, as it has been stated in Theorem 4 of Section 4.2 .

Case 2B: For $a_{1}=\frac{m}{2}, \alpha=0, \beta=4, \gamma=1, \eta=0$, we have:

$$
\begin{aligned}
y_{n} & =x_{n}-m \frac{2 f\left(x_{n}\right)}{2 f^{\prime}\left(x_{n}\right)+m f\left(x_{n}\right)}, \\
x_{n+1} & =y_{n}-m u_{n} \frac{1+4 u_{n}}{\left(1+u_{n}\right)^{2}} \frac{f\left(x_{n}\right)}{f^{\prime}\left(x_{n}\right)+m f\left(x_{n}\right)} .
\end{aligned}
$$

This selection of the values of the parameters give an iterative method with the same stability properties as in the previous case. 


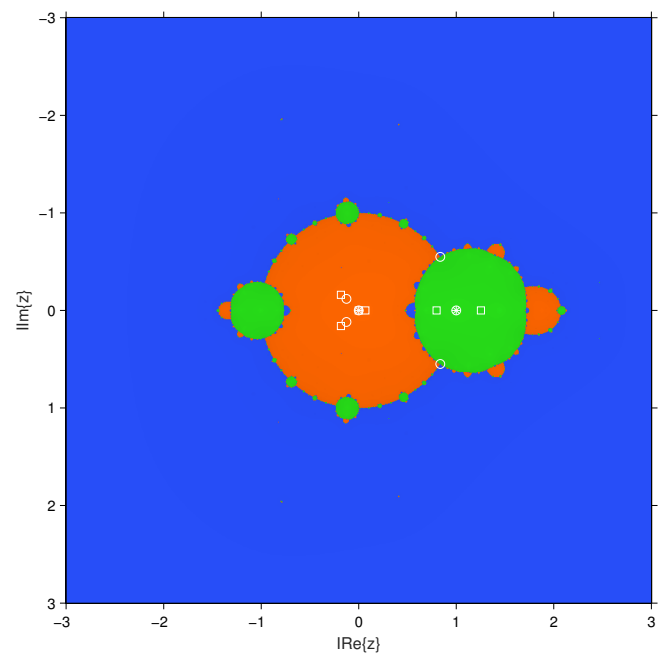

(a) $\eta=-75$

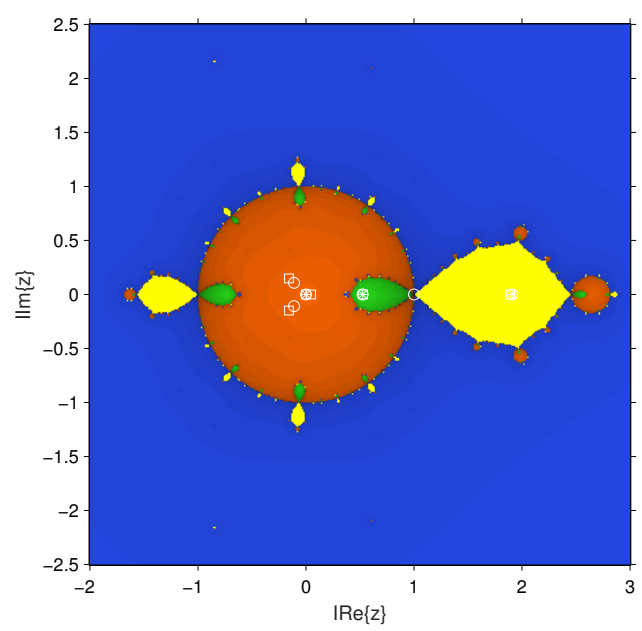

(c) $\eta=-120$

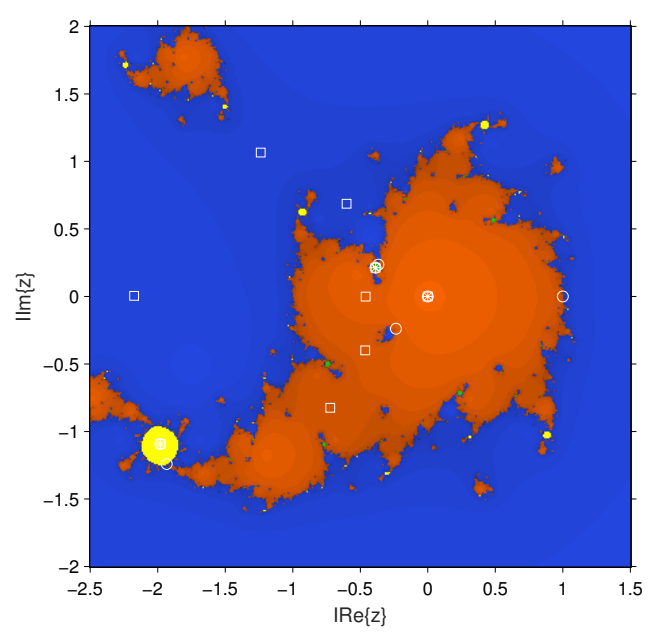

(b) $\eta=0.938074+1.66811 i$

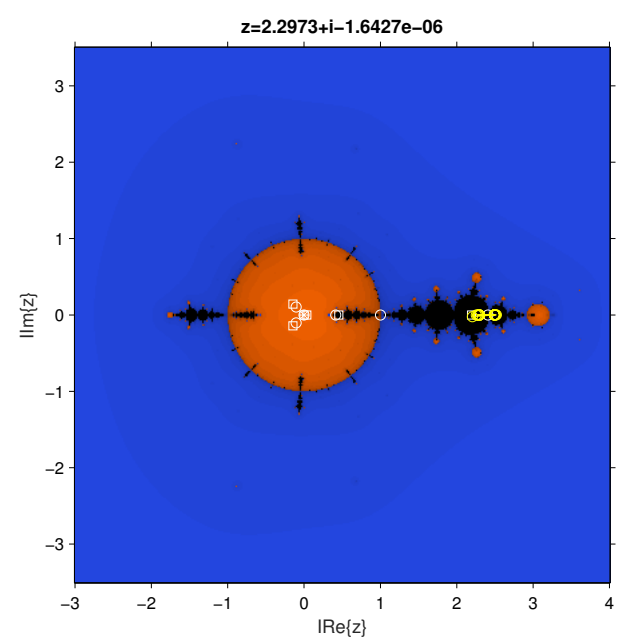

(d) $\eta=-150$

Fig. 11: Dynamical planes corresponding to unstable performance of $s(z, \eta)$

Case 2C: For $a_{1}=0, \alpha=0, \beta=2, \gamma=1, \eta=-44$, we have:

$$
\begin{aligned}
y_{n} & =x_{n}-m \frac{f\left(x_{n}\right)}{f^{\prime}\left(x_{n}\right)}, \\
x_{n+1} & =y_{n}-m u_{n} \frac{1+2 u_{n}}{1+u_{n}^{2}-44 u_{n}^{3}} \frac{f\left(x_{n}\right)}{f^{\prime}\left(x_{n}\right)} .
\end{aligned}
$$

As $a_{1}=0$ and $\eta=-44$, Theorem 5 in Section 4.2 sets that $z=1$ is not an strange fixed point; so, divergence is avoided. Indeed, Proposition 2 states that the strange fixed points are simultaneously repulsive for these values of the parameters and Theorem 7 says that there exist four independent free critical points and in Figure 9 it can be seen that $\eta=-44$ is in the stable area of all of them.

Case 2D: For $a_{1}=0, \alpha=1, \beta=2, \gamma=1, \eta=-44$, we have:

$$
\begin{aligned}
y_{n} & =x_{n}-m \frac{f\left(x_{n}\right)}{f^{\prime}\left(x_{n}\right)}, \\
x_{n+1} & =y_{n}-m u_{n} \frac{1+2 u_{n}+u_{n}^{2}}{1+u_{n}^{2}-44 u_{n}^{3}} \frac{f\left(x_{n}\right)}{f^{\prime}\left(x_{n}\right)} .
\end{aligned}
$$

As the values of $a_{1}$ and $\eta$ coincide in this case with those of Case $2 \mathrm{C}$, we can affirm that their qualitative behavior is the same. 
We compare the methods with the recent parametric-based optimal fourth order methods proposed by Behl et al. [18] (Behl) given by (28) and Lee et al. [19] (Lee) method.

In the comparison tables, we display the number of iteration indexes $n$, error at each iterations $\left|x_{n}-\xi\right|$, functional value at $x_{n},\left|f\left(x_{n}\right)\right|$, asymptotic error constant $\left|e_{n} / e_{n-1}^{4}\right|$, computational order of convergence $\rho_{n}$ using the formula by Jay [32] as:

$$
\rho_{n} \approx \frac{\ln \left|f\left(x_{n+1}\right) / f\left(x_{n}\right)\right|}{\ln \left|f\left(x_{n}\right) / f\left(x_{n-1}\right)\right|} .
$$

In this regard, we consider the test functions that have applications in real world problems as follows:

Example 1 Malthusian Model with Immigration

Let us consider the Malthusian population growth model with constant immigration. Let $\mathrm{P}(\mathrm{t})$ be population at any time $\mathrm{t}$, $\mathrm{r}$ is the fixed immigration, $\mathrm{k}$ is the constant birth rate, then we have:

$$
\frac{d P}{d t}=k P(t)+r, P(0)=P_{0}
$$

The solution is given as

$$
P(t)=P_{0} e^{k t}+\frac{r}{k}\left(e^{k t}-1\right) .
$$

Suppose, a certain community has 1000 thousand individuals initially and 300 thousand immigrate into the community in the first year. Also, let there be 1365 thousand people at the end of one year. Using the data, we are required to solve the non-linear equation:

$$
f_{1}(x)=1365-1000 e^{x}-\frac{300}{x}\left(e^{x}-1\right)
$$

to find the birth rate. The desired zero of $f_{1}$ is 0.05504622451335177827483421 with $\mathrm{m}=1$. We choose $x_{0}=0.06$.

Example 2 We consider a standard nonlinear test function as follows:

$$
f_{2}(x)=(x-1)^{2}\left(2 x^{2}-2 \sin \frac{\pi x}{6}-\sqrt{x}\right)^{2}
$$

It has a multiple zero $\xi=1$ of multiplicity of order four We consider an initial guess $x_{0}=3.3$ and $m=4$ for this problem.

Example 3 We consider another standard nonlinear test function as follows:

$$
f_{3}(x)=\left(e^{x}+x-20\right)^{2}
$$

It has a multiple zero $\xi=2.842438953$ of multiplicity of order two We consider an initial guess $x_{0}=3.0$ and $m=2$ for this problem.

\section{Example 4 Eigenvalue problem:}

One of the challenging task of linear algebra is to find the eigenvalues of a large square matrix. It gets complicated further if we are required to find the zeros of characteristic equation of a square matrix of order greater than 4 . Let us consider the following square matrix of order 4

$$
A=\frac{1}{8}\left[\begin{array}{ccccccccc}
-12 & 0 & 0 & 19 & -19 & 76 & -19 & 18 & 437 \\
-64 & 24 & 0 & -24 & 24 & 64 & -8 & 32 & 376 \\
-16 & 0 & 24 & 4 & -4 & 16 & -4 & 8 & 92 \\
-40 & 0 & 0 & -10 & 50 & 40 & 2 & 20 & 242 \\
-4 & 0 & 0 & -1 & 41 & 4 & 1 & 2 & 25 \\
-40 & 0 & 0 & 18 & -18 & 104 & -18 & 20 & 462 \\
-84 & 0 & 0 & -29 & 29 & 84 & 21 & 42 & 501 \\
16 & 0 & 0 & -4 & 4 & -16 & 4 & 16 & -92 \\
0 & 0 & 0 & 0 & 0 & 0 & 0 & 0 & 24
\end{array}\right]
$$

The corresponding characteristic polynomial of the above matrix (A) is given as follows:

$$
f_{4}(x)=x^{9}-29 x^{8}+349 x^{7}-2261 x^{6}+8455 x^{5}-17663 x^{4}+15927 x^{3}+6993 x^{2}-24732 x+12960 .
$$

The above function has one multiple zero at $\xi=3$ of multiplicity 4 with initial approximation $x_{0}=2.4$. 
Example 5 Fractional conversion in a chemical reactor:

Let us consider the following non-linear function involved in fractional conversion in a chemical reactor (See [2])

$$
f_{5}(x)=\frac{x}{1-x}-5 \log \left[\frac{0.4(1-x)}{0.4-0.5 x}\right]+4.45977 .
$$

Here $x$ represents the fractional conversion of species $\mathrm{X}$ in a chemical reactor. It is evident that there will be no physical meaning of above fractional conversion if $x$ is less than zero or greater than one. Due to which we take $x$ in the bounded region $0 \leq x \leq 1$. The required solution is $\xi=0.757396246253753879459641297929$. Moreover, we consider the initial guess as $x_{0}=0.76$.

We have done our calculations with 1000 significant digits to minimize the round off error. Due to limitations, we display the values up to 10 significant digits only with exponent power denoted by $E(-i)$ for $E \times 10^{-i}$ in the Tables $1-5$. D stands for divergence.

\begin{tabular}{|c|c|c|c|c|c|c|}
\hline Schemes & $n$ & $x_{n}$ & $\left|f\left(x_{n}\right)\right|$ & $\left|x_{n}-\xi\right|$ & $\left|e_{n} / e_{n-1}^{4}\right|$ & $\rho_{n}$ \\
\hline \multirow[t]{3}{*}{ Behl } & 1 & 0.05504622471 & $2.4070009598(-7)$ & $1.985631340(-10)$ & $3.297261107(-1)$ & \multirow[b]{3}{*}{3.999999999} \\
\hline & 2 & 0.05504622451 & $6.256736895(-37)$ & $5.161432453(-40)$ & $3.320288127(-1)$ & \\
\hline & 3 & 0.05504622451 & $2.856501193(-155)$ & $2.356442057(-158)$ & $3.320288127(-1)$ & \\
\hline \multirow[t]{3}{*}{ Lee } & 1 & 0.05504622479 & $3.431024134(-7)$ & $2.830389004(-10)$ & $4.700032375(-1)$ & \multirow[b]{3}{*}{3.999999999} \\
\hline & 2 & 0.05504622451 & $3.688395710(-36)$ & $3.042705109(-39)$ & $4.741058891(-1)$ & \\
\hline & 3 & 0.05504622451 & $4.925977797(-152)$ & $4.063636060(-155)$ & $4.741058893(-1)$ & \\
\hline \multirow[t]{3}{*}{$S 1 A$} & 1 & 0.05504622443 & $9.146728958(-8)$ & $7.545502468(-11)$ & $1.252976386(-1)$ & \multirow[b]{3}{*}{4.000000000} \\
\hline & 2 & 0.05504622451 & $5.092442840(-39)$ & $4.200959730(-42)$ & $1.295972709(-1)$ & \\
\hline & 3 & 0.05504622451 & $4.892913910(-164)$ & $4.036360338(-167)$ & $1.295972708(-1)$ & \\
\hline \multirow[t]{3}{*}{$S 1 B$} & 1 & 0.05504622332 & $1.444440637(-6)$ & $1.191576841(-9)$ & 1.978685516 & \multirow[b]{3}{*}{4.000000000} \\
\hline & 2 & 0.05504622451 & $5.031003323(-33)$ & $4.150275816(-36)$ & 2.058679519 & \\
\hline & 3 & 0.05504622451 & $7.404133206(-139)$ & $6.107965551(-142)$ & 2.058679500 & \\
\hline \multirow[t]{3}{*}{$S 2 A$} & 1 & 0.05504622512 & $7.366771663(-7)$ & $6.077144519(-10)$ & 1.009146655 & \multirow[b]{3}{*}{3.999999999} \\
\hline & 2 & 0.05504622451 & $1.691299495(-34)$ & $1.395220583(-37)$ & 1.022926942 & \\
\hline & 3 & 0.05504622451 & $4.698873591(-145)$ & $3.876288719(-148)$ & 1.022926944 & \\
\hline \multirow[t]{3}{*}{$S 2 B$} & 1 & 0.05504622906 & $5.521846244(-6)$ & $4.555191759(-9)$ & 7.564171818 & \multirow[b]{3}{*}{3.999999999} \\
\hline & 2 & 0.05504622451 & $4.064486249(-30)$ & $3.352957233(-33)$ & 7.787559135 & \\
\hline & 3 & 0.05504622451 & $1.193139078(-126)$ & $9.842681359(-130)$ & 7.787559344 & \\
\hline \multirow[t]{3}{*}{$S 2 C$} & 1 & 0.05504622485 & $4.142353002(-7)$ & $3.417192631(-10)$ & $5.674455339(-1)$ & \multirow[b]{3}{*}{3.999999999} \\
\hline & 2 & 0.05504622451 & $9.650986863(-36)$ & $7.961484978(-39)$ & $5.838700991(-1)$ & \\
\hline & 3 & 0.05504622451 & $2.843611096(-150)$ & $2.345808501(-153)$ & $5.838701003(-1)$ & \\
\hline \multirow[t]{3}{*}{$S 2 D$} & 1 & 0.05504622478 & $3.352230221(-7)$ & $2.765388755(-10)$ & $4.592095523(-1)$ & \multirow[b]{3}{*}{3.999999999} \\
\hline & 2 & 0.05504622451 & $3.361071599(-36)$ & $2.772682362(-39)$ & $4.741058885(-1)$ & \\
\hline & 3 & 0.05504622451 & $3.396670923(-152)$ & $2.802049667(-155)$ & $4.741058893(-1)$ & \\
\hline
\end{tabular}

Table 1: Comparison of methods for $f_{1}(x)$

It can be observed in the Tables 1-5 that proposed methods behave as it is expected, confirming the theoretical results. It is also seen that methods coming from stable values of the dynamical analysis perform better than those classified as unstable, that is, methods S1A and S2A have lower errors than S1B and S2B. Moreover, both S2C and S2D corresponding to stable values for rational weight functions show very good results. In general, schemes with better results are S2D and S1A.

\section{Conclusions}

In this manuscript, we have designed a new optimal fourth-order class of iterative methods for multiple roots that contains some known methods. As it has been done by using weight-function procedure, a big variety of subclasses can be defined holding the order of convergence. We have selected some polynomial and rational weight functions to analyze the dynamical behavior of the resulting families. Some interesting results have been obtained that have been confirmed by numerical tests.

Acknowledgement: The authors would like to thank the anonymous reviewers for their help to improve the final version of this manuscript.

\section{Conflicts of Interest}

The authors declare that there are no conflicts of interest regarding the publication of this paper. 


\begin{tabular}{|lll|l|l|l|l|}
\hline Schemes & $n$ & $x_{n}$ & $\left|f\left(x_{n}\right)\right|$ & $\left|x_{n}-\xi\right|$ & $\left|e_{n} / e_{n-1}^{4}\right|$ & $\rho_{n}$ \\
\hline Behl & 1 & 1.163143822 & $6.213998677(-3)$ & $1.631438226(-1)$ & $5.829875630(-3)$ & \\
& 2 & 1.000157615 & $4.151009251(-15)$ & $1.576154954(-4)$ & $2.224930119(-1)$ & \\
& 3 & 1.000000000 & $2.514716599(-62)$ & $2.4729344401(-16)$ & $4.006977989(-1)$ & 3.878178342 \\
\hline Lee & 1 & 1.164331487 & $6.408520313(-3)$ & $1.643314873(-1)$ & $5.872316329(-3)$ & \\
& 2 & 1.000177229 & $6.636208744(-15)$ & $1.772298136(-4)$ & $2.430265022(-1)$ & \\
& 3 & 1.000000000 & $2.798865388(-61)$ & $4.516851824(-16)$ & $4.578136319(-1)$ & 3.869467328 \\
\hline$S 1 A$ & 1 & 1.111018298 & $1.228691791(-3)$ & $1.110182987(-1)$ & $3.967192040(-3)$ & \\
& 2 & 1.000006141 & $9.563645886(-21)$ & $6.141084161(-6)$ & $4.042655903(-2)$ & \\
& 3 & 1.000000000 & $2.732822485(-94)$ & $2.524894267(-24)$ & $1.775264216(-3)$ & 4.298602378 \\
\hline$S 1 B$ & 1 & 1.258896314 & $4.537016137(-2)$ & $2.588963144(-1)$ & $9.251550503(-3)$ & \\
& 2 & 0.999300233 & $1.610347850(-12)$ & $6.997660387(-4)$ & $1.557576511(-1)$ & \\
& 3 & 1.000000918 & $4.794357138(-24)$ & $9.189095440(-7)$ & $3.832316038(6)$ & 2.283295522 \\
\hline$S 2 A$ & 1 & 1.201747543 & $1.539961374(-2)$ & $2.017475436(-1)$ & $7.209363302(-3)$ & \\
& 2 & 1.000582286 & $7.737953036(-13)$ & $5.822863250(-3)$ & $3.514823879(-1)$ & \\
& 3 & 1.000000000 & $6.739694875(-52)$ & $1.000576732(-13)$ & $8.703702461(-1)$ & 3.792642663 \\
\hline$S 2 B$ & 1 & 1.944480972 & $1.778648632(1)$ & $9.444809728(-1)$ & $3.375062885(-2)$ & \\
& 2 & 1.186510886 & $1.099612324(-2)$ & $1.865108863(-1)$ & $2.343858195(-1)$ & \\
& 3 & 1.002879056 & $4.643207582(-10)$ & $2.879056528(-3)$ & 2.379213680 & 2.298150490 \\
\hline$S 2 C$ & 1 & 1.049813740 & $4.507825712(-5)$ & $4.981374096(-2)$ & $1.780073004(-3)$ & \\
& 2 & 1.000002343 & $2.026524674(-22)$ & $2.343030116(-6)$ & $3.805232861(-1)$ & \\
& 3 & 1.000000000 & $4.756664815(-91)$ & $1.630857360(-23)$ & $5.411335917(-1)$ & 3.956222856 \\
\hline$S 2 D$ & 1 & 1.03744627 & $1.409885742(-4)$ & $3.744627423(-2)$ & $1.338126801(-3)$ & \\
& 2 & 1.000000682 & $1.456074038(-24)$ & $6.821597270(-7)$ & $3.469378014(-1)$ & \\
& 3 & 1.000000000 & $6.514566551(-100)$ & $9.921143288(-26)$ & $4.581602608(-1)$ & 3.968676714 \\
\hline
\end{tabular}

Table 2: Comparison of methods for $f_{2}(x)$

\begin{tabular}{|lll|l|l|l|l|}
\hline Schemes & $n$ & $x_{n}$ & $\left|f\left(x_{n}\right)\right|$ & $\left|x_{n}-\xi\right|$ & $\left|e_{n} / e_{n-1}^{4}\right|$ & $\rho_{n}$ \\
\hline Behl & 1 & $D$ & - & - & - & - \\
& 2 & - & - & - & - & - \\
& 3 & - & - & - & - & - \\
\hline Lee & 1 & 2.842655376 & $1.544574100(-5)$ & $2.164225216(-4)$ & $3.511616083(-1)$ & \\
& 2 & 2.842438953 & $3.252744931(-28)$ & $9.932703953(-16)$ & $4.527491873(-1)$ & \\
& 3 & 2.842438953 & $6.407431039(-119)$ & $4.408435159(-61)$ & $4.529126925(-1)$ & 3.999970502 \\
\hline$S 1 A$ & 1 & 2.842419561 & $1.239841123(-7)$ & $1.939230730(-5)$ & $3.146545826(-2)$ & \\
& 2 & 2.842438954 & $4.163414480(-17)$ & $3.553589935(-10)$ & $2.512750523(9)$ & \\
& 3 & 2.842438953 & $1.355227554(-75)$ & $2.027442745(-39)$ & $1.271391647(-1)$ & 6.173522651 \\
\hline$S 1 B$ & 1 & 2.842092176 & $3.963444678(-5)$ & $3.467768038(-4)$ & $5.626711084(-1)$ & \\
& 2 & 2.842439187 & $1.807060181(-11)$ & $2.341147341(-7)$ & $1.618931931(7)$ & \\
& 3 & 2.842438953 & $1.227939872(-50)$ & $6.102826703(-27)$ & $2.031496362(13)$ & 6.176813861 \\
\hline$S 2 A$ & 1 & 2.842848889 & $5.542617408(-5)$ & $4.099357747(-4)$ & $6.651512275(-1)$ & \\
& 2 & 2.842438953 & $2.520918726(-25)$ & $2.765170463(-14)$ & $9.791711911(-1)$ & \\
& 3 & 2.842438953 & $1.082800758(-106)$ & $5.730819932(-55)$ & $9.802325631(-1)$ & 3.999920660 \\
\hline$S 2 B$ & 1 & 2.844556510 & $1.481339170(-3)$ & $2.117557214(-3)$ & 3.43589378 & \\
& 2 & 2.842438953 & $7.560308189(-18)$ & $1.514301096(-10)$ & 7.531329058 & \\
& 3 & 2.842438953 & $5.299869283(-75)$ & $4.009360119(-39)$ & 7.624758960 & 3.999007446 \\
\hline$S 2 C$ & 1 & 2.842561204 & $4.928005506(-6)$ & $1.222511616(-4)$ & $1.983615854(-1)$ & \\
& 2 & 2.842438953 & $5.121499337(-30)$ & $1.246352954(-16)$ & $5.579934095(-1)$ & \\
& 3 & 2.842438953 & $5.985465465(-126)$ & $1.347384204(-64)$ & $5.583766667(-1)$ & 3.999966765 \\
\hline$S 2 D$ & 1 & 2.842517962 & $2.058262941(-6)$ & $7.900902501(-5)$ & $1.281980085(-1)$ & \\
& 2 & 2.842438953 & $1.025949521(-31)$ & $1.764028038(-17)$ & $4.526876781(-1)$ & \\
& 3 & 2.842438953 & $6.341439870(-133)$ & $4.385674810(-68)$ & $9.802325631(-1)$ & 3.999977815 \\
\hline
\end{tabular}

Table 3: Comparison of methods for $f_{3}(x)$

\section{References}

1. Constantinides A., Mostoufi N.: Numerical Methods for Chemical Engineers with MATLAB Applications, Prentice Hall PTR, New Jersey, 1999.

2. Shacham M.: Numerical solution of constrained nonlinear algebraic equations, Int. Numer. Method Eng. 23 (1986) $1455-1481$.

3. Hueso J.L., Martínez E., Teruel C.: Determination of multiple roots of nonlinear equations and applications, Math. Chem. 53 (2015) 880-892.

4. Anza S., Vicente C., Gimeno B., Boria V.E., Armendá riz J.:, Long-term multipactor discharge in multicarrier systems, Physics of Plasmas 14(8) (2007) $082-112$.

5. Neta B., Johnson A.N.: High order nonlinear solver for multiple roots, Comp. Math. Appl. 55(9) (2008) $2012-2017$.

6. Li S., Liao X., Cheng L.: A new fourth-order iterative method for finding multiple roots of nonlinear equations, Appl. Math. Comput. 215 (2009) $1288-1292$.

7. Neta B., Extension of Murakami's high-order non-linear solver to multiple roots, Int. J. Comput. Math. 87(5) (2010) 1023-1031.

8. Sharma J.R., Sharma R., Modified Jarratt method for computing multiple roots, Appl. Math. Comput. 217 (2010) $878-881$.

9. Li S.G., Cheng L.Z., Neta B.: Some fourth-order nonlinear solvers with closed formulae for multiple roots, Comput. Math. Appl. 59 (2010) 126-135. 


\begin{tabular}{|c|c|c|c|c|c|c|}
\hline Schemes & $n$ & $x_{n}$ & $\left|f\left(x_{n}\right)\right|$ & $\left|x_{n}-\xi\right|$ & $\left|e_{n} / e_{n-1}^{4}\right|$ & $\rho_{n}$ \\
\hline \multirow[t]{3}{*}{ Behl } & 1 & 2.998959565 & $9.383771848(-11)$ & $1.040434253(-3)$ & $8.028042081(-3)$ & \multirow[b]{3}{*}{3.999769820} \\
\hline & 2 & 2.999999999 & $1.184831738(-49)$ & $1.961741019(-13)$ & $1.674106588(-1)$ & \\
\hline & 3 & 2.999999999 & $3.074166125(-205)$ & $2.489770611(-52)$ & $1.681096500(-1)$ & \\
\hline \multirow[t]{3}{*}{ Lee } & 1 & 2.999125476 & $4.683130806(-11)$ & $8.745234093(-4)$ & $6.747865812(-3)$ & \multirow[b]{3}{*}{3.999790187} \\
\hline & 2 & 2.999999999 & $1.246746011(-51)$ & $6.283071474(-14)$ & $1.074202709(-1)$ & \\
\hline & 3 & 2.999999999 & $6.386425623(-214)$ & $1.680900351(-54)$ & $1.078583984(-1)$ & \\
\hline \multirow[t]{3}{*}{$S 1 A$} & 1 & 2.999171531 & $3.771693353(-11)$ & $8.284688897(-4)$ & $6.392506865(-3)$ & \multirow[b]{3}{*}{3.999961079} \\
\hline & 2 & 2.999999999 & $5.372557156(-54)$ & $1.609803118(-14)$ & $3.417180706(-2)$ & \\
\hline & 3 & 2.999999999 & $2.220379952(-225)$ & $2.295272892(-57)$ & $3.417773437(-2)$ & \\
\hline \multirow[t]{3}{*}{$S 1 B$} & 1 & $D$ & - & - & - & - \\
\hline & 2 & - & - & - & - & - \\
\hline & 3 & - & - & - & - & - \\
\hline \multirow[t]{3}{*}{$S 2 A$} & 1 & 2.999085183 & $5.607940939(-11)$ & $9.148166811(-4)$ & $7.058770687(-3)$ & \multirow[b]{3}{*}{3.999736884} \\
\hline & 2 & 2.999999999 & $1.762468454(-50)$ & $1.218310091(-13)$ & $1.739488416(-1)$ & \\
\hline & 3 & 2.999999999 & $1.761111249(-208)$ & $3.851892880(-53)$ & $1.748408203(-1)$ & \\
\hline \multirow[t]{3}{*}{$S 2 B$} & 1 & $D$ & - & - & - & - \\
\hline & 2 & - & - & - & - & - \\
\hline & 3 & - & - & - & - & - \\
\hline \multirow[t]{3}{*}{$S 2 C$} & 1 & 2.999126080 & $4.670203793(-11)$ & $8.739194142(-4)$ & $6.743205356(-3)$ & \multirow[b]{3}{*}{3.999739394} \\
\hline & 2 & 2.999999999 & $1.960414149(-51)$ & $7.035815790(-14)$ & $1.206226626(-1)$ & \\
\hline & 3 & 2.999999999 & $6.236175057(-213)$ & $2.971372350(-54)$ & $1.212548828(-1)$ & \\
\hline \multirow[t]{3}{*}{$S 2 D$} & 1 & 2.999134454 & $4.493725036(-11)$ & $8.655455253(-4)$ & $6.678592016(-3)$ & \multirow[b]{3}{*}{3.999746898} \\
\hline & 2 & 2.999999999 & $1.052660468(-51)$ & $6.022815808(-14)$ & $1.073099319(-1)$ & \\
\hline & 3 & 2.999999999 & $3.245631148(-214)$ & $1.419228402(-54)$ & $1.078583984(-1)$ & \\
\hline
\end{tabular}

Table 4: Comparison of methods for $f_{4}(x)$

\begin{tabular}{|c|c|c|c|c|c|c|}
\hline Schemes & $n$ & $x_{n}$ & $\left|f\left(x_{n}\right)\right|$ & $\left|x_{n}-\xi\right|$ & $\left|e_{n} / e_{n-1}^{4}\right|$ & $\rho_{n}$ \\
\hline \multirow[t]{3}{*}{ Behl } & 1 & 0.7573967298 & $3.857041135(-5)$ & $4.835750028(-7)$ & $1.052117421(4)$ & \multirow[b]{3}{*}{3.999998886} \\
\hline & 2 & 0.7573962462 & $4.773340820(-20)$ & $5.984603017(-22)$ & $1.094409804(4)$ & \\
\hline & 3 & 0.7573962462 & $1.119725925(-79)$ & $1.403862704(-81)$ & $1.094418051(4)$ & \\
\hline \multirow[t]{3}{*}{ Lee } & 1 & 0.7573969208 & $5.380728772(-5)$ & $6.746046923(-7)$ & $1.467742015(4)$ & \multirow[b]{3}{*}{3.999998137} \\
\hline & 2 & 0.7573962462 & $2.599221296(-19)$ & $3.258788383(-21)$ & $1.573470948(4)$ & \\
\hline & 3 & 0.7573962462 & $1.415397885(-76)$ & $1.774563094(-78)$ & $1.573500217(4)$ & \\
\hline \multirow[t]{3}{*}{$S 1 A$} & 1 & 0.7573960528 & $15425561203(-5)$ & $1.93399438(-7)$ & $4.207804721(3)$ & \multirow[b]{3}{*}{4.000001025} \\
\hline & 2 & 0.7573962462 & $6.916822161(-22)$ & $8.672004855(-24)$ & $6.198672348(3)$ & \\
\hline & 3 & 0.7573962462 & $2.796098011(-87)$ & $3.505623676(-89)$ & $6.198509111(3)$ & \\
\hline \multirow[t]{3}{*}{$S 1 B$} & 1 & 0.7573960323 & $1.706379158(-5)$ & $2.139389766(-7)$ & $4.654684859(3)$ & \multirow[b]{3}{*}{4.000001170} \\
\hline & 2 & 0.7573962462 & $1.157469114(-21)$ & $1.451183440(-23)$ & $6.927278652(3)$ & \\
\hline & 3 & 0.7573962462 & $2.450337898(-86)$ & $3.072124981(-88)$ & $6.927070760(3)$ & \\
\hline \multirow[t]{3}{*}{$S 2 A$} & 1 & 0.7573976622 & $1.129449059(-4)$ & $1.416021340(-6)$ & $3.080847256(4)$ & \multirow[b]{3}{*}{3.999994210} \\
\hline & 2 & 0.7573962462 & $1.143901592(-17)$ & $1.434173082(-19)$ & $3.567158226(4)$ & \\
\hline & 3 & 0.7573962462 & $1.203793421(-69)$ & $1.509262802(-71)$ & $3.567455789(4)$ & \\
\hline \multirow[t]{3}{*}{$S 2 B$} & 1 & 0.7573977792 & $1.2227772442(-4)$ & $1533026607(-6)$ & $3.335416411(4)$ & \multirow[b]{3}{*}{3.999993438} \\
\hline & 2 & 0.7573962462 & $1.719392607(-17)$ & $2.155698194(-19)$ & $3.902915851(4)$ & \\
\hline & 3 & 0.7573962462 & $6.723109888(-69)$ & $8.429136999(-71)$ & $3.903294151(4)$ & \\
\hline \multirow[t]{3}{*}{$S 2 C$} & 1 & 0.7573967924 & $4.356152665(-5)$ & $5.461503708(-7)$ & $1.188263074(4)$ & \multirow[b]{3}{*}{3.999996205} \\
\hline & 2 & 0.7573962462 & $1.399481427(-19)$ & $1.754607744(-21)$ & $1.972109887(4)$ & \\
\hline & 3 & 0.7573962462 & $1.491002361(-77)$ & $1.869352633(-79)$ & $1.972291331(4)$ & \\
\hline \multirow[t]{3}{*}{$S 2 D$} & 1 & 0.7573966343 & $3.095858328(-5)$ & $3.881425976(-7)$ & $8.444844883(3)$ & \multirow[b]{3}{*}{3.999997087} \\
\hline & 2 & 0.7573962462 & $2.848301990(-20)$ & $3.571074709(-22)$ & $1.573380284(4)$ & \\
\hline & 3 & 0.7573962462 & $2.041031224(-80)$ & $2.558954427(-82)$ & $1.573500217(4)$ & \\
\hline
\end{tabular}

Table 5: Comparison of methods for $f_{5}(x)$

10. Zhou X., Chen X., Song Y.: Constructing higher-order methods for obtaining the muliplte roots of nonlinear equations, Comput. Math. Appl. 235 (2011) 4199-4206.

11. Sharifi M., Babajee D.K.R., Soleymani F.: Finding the solution of nonlinear equations by a class of optimal methods, Comput. Math. Appl. 63 (2012) 764-774.

12. Soleymani F., Babajee D.K.R., Lofti T.: On a numerical technique for finding multiple zeros and its dynamic, Egypt. Math. Soc. 21 (2013) $346-353$.

13. Soleymani F., Babajee D.K.R., Computing multiple zeros using a class of quartically convergent methods, Alex. Eng. J. 52 (2013) 531-541.

14. Liu B., Zhou X., A new family of fourth-order methods for multiple roots of nonlinear equations, Non. Anal. Model. Cont. 18(2) (2013) 143-152.

15. Zhou X., Chen X., Song Y., Families of third and fourth order methods for multiple roots of nonlinear equations, Appl. Math. Comput. 219 (2013) $6030-6038$.

16. Thukral R., A new family of fourth-order iterative methods for solving nonlinear equations with multiple roots, Numer. Math. Stoch. 6 (1) (2014) 37-44.

17. Behl R., Cordero A., Motsa S.S., Torregrosa J.R.: On developing fourth-order optimal families of methods for multiple roots and their dynamics, Appl. Math. Comput. 265(15) (2015) 520-532. 
18. Behl R., Cordero A., Motsa S.S., Torregrosa J.R., Kanwar V.: An optimal fourth-order family of methods for multiple roots and its dynamics, Numer. Algor. 71(4) (2016) 775-796.

19. Lee M.Y., Kim Y.I., Magreñán Á. A.: On the dynamics of tri-parametric family of optimal fourth-order multiple-zero finders with a weight function of the principal $\mathrm{m}^{\text {th }}$ root of a function-function ratio, Appl. Math. Comput. 315 (2017) 564-590.

20. Kim Y.I., Geum Y-H.: A triparametric family of optimal fourth-order multiple-root finders and their Dynamics, Discrete Dynamics in Nature and Society 2016 (2016) Article ID 843675923 pages.

21. Kim Y.I., Geum Y-H.: A two-parametric family of fourth-order iterative methods with optimal convergence for multiple zeros, J. Appl. Math. 2013 (2013) Article ID 3690677 pages.

22. Kim Y.I., Geum Y-H.: A new biparametric family of two-point optimal fourth-order multiple-root finders, J. Appl. Math. 2014 (2014) Article ID 737305 7 pages.

23. Amat S., Argyros I.K., Busquier S., Magreñán Á. A.: Local convergence and the dynamics of a two-point four parameter Jarratt-like method under weak conditions, Numer. Algor. (2017), doi: 10.1007/s11075-016-0152-5.

24. Cordero A., García-Maimó J., Torregrosa J.R., Vassileva M.P., Vindel P.: Chaos in King's iterative family, Appl. Math. Lett. 26 (2013) $842-848$.

25. Geum Y.H., Kim Y.I., Neta B.: A sixth-order family of three-point modified Newton-like multiple-root finders and the dynamics behind their extraneous fixed points, Appl. Math. Comput. 283 (2016) 120-140.

26. Amiri A., Cordero A., Darvishi M.T., Torregrosa J.R.: Stability analysis of a parametric family of seventh-order iterative methods for solving nonlinear systems, Appl. Math. Comput. 323 (2018) 43-57.

27. Blanchard P.: The dinamics of Newton's method, Proc, Symp. Appl. math. 49 (1994) 139-154.

28. Beardon A.F.: Iteration of rational functions: Complex and Analytic Dynamical Systems, Graduate Texts in Mathematics, 132, Springer-Verlag New York, 1991.

29. Devaney R.L.: An Introduction to Chaotic Dynamical Systems, Addison-Wesley Publishing Company, 1989.

30. Blanchard P.: Complex analytic dynamics on the Riemann sphere, Bull. AMS 11 (1) (1984) 85-141.

31. Chicharro F., Cordero A., Torregrosa J.R.: Drawing dynamical and parameter planes of iterative families and methods, Sci. World 2013 (2013) Article ID 780153, 11 pages.

32. Jay L.O.: A note on Q-order of convergence. BIT Numer. Math. 41 (2001) 422-429. 